\title{
Correlations between peripheral blood biomarkers and clinical outcomes in advanced non-small cell lung cancer patients who received immunotherapy-based treatments
}

\author{
Yuequan Shi ${ }^{1} \wedge$, Xiaoyan Liu ${ }^{1}$, Jia Liu ${ }^{1}$, Dongming Zhang ${ }^{1}$, Xiangning Liu ${ }^{1}$, Yuan Yue ${ }^{1}$, Qing Zhou ${ }^{1}$, \\ Xiaoxing Gao', Minjiang Chen ${ }^{1}$, Yan Xu' ${ }^{1}$, Jing Zhao ${ }^{1}$, Wei Zhong ${ }^{1}$, Mariano Provencio ${ }^{2}$, Jacek Jassem ${ }^{3}$, \\ Terence M. Williams ${ }^{4}$, Andreas Seeber ${ }^{5}$, Florian Kocher ${ }^{5}$, Mengzhao Wang ${ }^{1}$
}

${ }^{1}$ Department of Respiratory and Critical Care Medicine, Peking Union Medical College Hospital, Chinese Academy of Medical Sciences \& Peking Union Medical College, Beijing, China; ${ }^{2}$ Medical Oncology Department, Hospital Universitario Puerta de Hierro Majadahonda, Madrid, Spain; ${ }^{3}$ Department of Oncology and Radiotherapy, Medical University of Gdańsk, Gdańsk, Poland; ${ }^{4}$ Department of Radiation Oncology, City of Hope National Medical Center, Duarte, CA, USA; ${ }^{5}$ Department of Internal Medicine V (Hematology and Oncology), Comprehensive Cancer Center Innsbruck, Medical University of Innsbruck, Innsbruck, Austria

Contributions: (I) Conception and design: M Wang, Y Shi; (II) Administrative support: None; (III) Provision of study materials or patients: Y Shi, XY Liu, J Liu, D Zhang, XN Liu, Y Yue, Q Zhou, X Gao, M Chen, Y Xu, J Zhao, W Zhong, M Wang; (IV) Collection and assembly of data: Y Shi, J Liu, D Zhang; (V) Data analysis and interpretation: Y Shi, XY Liu, M Wang; (VI) Manuscript writing: All authors; (VII) Final approval of manuscript: All authors.

Correspondence to: Mengzhao Wang. Department of Respiratory and Critical Care Medicine, Peking Union Medical College Hospital, Chinese Academy of Medical Sciences \& Peking Union Medical College, Beijing 100730, China. Email: mengzhaowang@sina.com.

Background: Peripheral blood-based biomarkers (PBB) predicting response, survival and immune-related adverse events (irAEs) in patients with advanced non-small cell lung cancer (NSCLC) treated with immune checkpoint inhibitors (ICIs) are still a matter of debate. Thus, we investigated the associations between PBB, the efficacy of ICIs and the incidence of irAEs.

Methods: Patients with advanced NSCLC, who had been treated at Peking Union Medical College Hospital and received ICIs or chemoimmunotherapy from January 2015 to December 2020, were retrospectively identified. $\mathrm{PBB}$ results were retrieved from medical records. Associations with overall response rate, survival, and incidence of irAEs were assessed using Kruskal-Wallis, Kaplan-Meier analysis, Pearson's chi-squared and Student's $t$-tests as required. Cox proportional hazards and logistic regression models were used to determine independent risk factors. Analyses were performed on the whole population $(\mathrm{n}=103)$, patients receiving ICIs only $(\mathrm{n}=32)$, and patients receiving chemoimmunotherapy $(\mathrm{n}=71)$. Changes in pretreatment and on-treatment PBB were also analyzed.

Results: Among 103 patients, 38 (36.9\%) developed irAEs. Pretreatment absolute lymphocyte count (ALC) was related to an increased risk of irAEs in the whole population [odds ratio (OR), 2.165; 95\% confidence interval (CI): 1.040 to $4.509, \mathrm{P}=0.039$ ] and patients receiving ICIs only (OR, 6.461; 95\% CI: 1.067 to 39.112; $\mathrm{P}=0.042)$. A low prognostic nutritional index ( $\mathrm{PNI} \leq 45)$ was associated with worse progression-free survival (PFS) and overall survival (OS) in the whole population, in patients receiving ICIs only, and in patients receiving chemoimmunotherapy. High pretreatment interleukin (IL)-6 was associated with both worse PFS and OS in the whole population (IL-6 $>13.80 \mathrm{pg} / \mathrm{mL}$ ), in patients receiving ICIs only (IL-6 $>11.30 \mathrm{pg} / \mathrm{mL}$ ), and in patients receiving chemoimmunotherapy (IL-6 $>11.85 \mathrm{pg} / \mathrm{mL}$ ). Increase of IL-6 during treatment was associated with inferior OS in the whole population $(\mathrm{P}<0.001)$.

Conclusions: Pretreatment ALC has the potential to predict irAEs in patients with advanced NSCLC treated with ICIs. Additionally, a low level of pretreatment PNI and high level of IL-6 may be associated

^ ORCID: 0000-0001-5469-8190. 
with shorter survival.

Keywords: Immune checkpoint inhibitors (ICIs); advanced non-small cell lung cancer (NSCLC); peripheral blood biomarker; immune-related adverse events (irAEs)

Submitted Jun 23, 2021. Accepted for publication Oct 02, 2021.

doi: $10.21037 /$ tlcr-21-710

View this article at: https://dx.doi.org/10.21037/tlcr-21-710

\section{Introduction}

Over the past decade, immune checkpoint inhibitors (ICIs) have emerged as a promising new treatment option in patients with cancer. ICIs, including anti-programmed cell death-1 (PD-1)/programmed death-ligand 1 (PD-L1), and anti-cytotoxic T-lymphocyte-associated antigen-4 (CTLA-4) antibodies, have shown durable efficacy in subsets of patients with lung cancer (1-4). Except for their significant clinical benefits, ICIs are associated with a specific spectrum of autoimmune and autoinflammatory side effects which are collectively termed as immune-related adverse events (irAEs). These toxicities can lead to significant morbidity, treatment discontinuation, diminished quality of life, and even fatal events have been described. It has been reported that the incidence of irAEs varied between $24 \%$ and $38 \%$ in non-small cell lung cancer (NSCLC) patients treated with immunotherapy-based treatment $(1,2,5-8)$. Thus, the identification of biomarkers predicting irAEs are desirable to render patients with increased susceptibility for ICI related toxicities. Previous studies have evaluated different serumbased biomarkers including interleukin (IL)-17, eosinophilia, and several cytokines. However, a sufficient tool predicting irAEs has yet to be determined (9-12).

Up to now, the most widely used stratification factors in clinical trials investigating ICIs are PD-L1 expression and microsatellite instability, since these factors have been associated with outcome $(13,14)$. However, long term responses have also been described in NSCLC patients lacking PD-(L)1 expression or microsatellite instability $(1,2)$. Peripheral blood biomarkers (PBB), including absolute blood cell counts (15) and calculated indexes, such as the neutrophil-to-lymphocyte ratio (NLR), and plateletto-lymphocyte ratio (PLR) have also been investigated as predictive biomarkers. Furthermore, the prognostic nutritional index (PNI) seems also to predict outcome upon ICIs treatment (16-18).

Cytokines are important regulators of host immune activity that promote the recruitment of immune cells into the tumor microenvironment (19) and may also serve as potential biomarkers. One of which is IL-6, which is a cytokine involved in immune regulation. Elevated level of IL-6 has been observed in various types of cancer such as melanoma (20), ovarian (21), and colorectal cancer (22), and it has been reported to play a role in tumor promotion by expansion and survival of malignant cells, neo-angiogenesis, and inflammation (23-26). In addition, monitoring longterm changes in peripheral blood cells and cytokine levels has been reported to facilitate the prediction of patient outcomes $(12,27,28)$. However, the relationships between the above-mentioned circulating parameters and ICIs outcomes are not well established. Moreover, published literature has rarely enrolled both blood cells and cytokines. Thus, there is still a quest towards novel and easy to use biomarkers with either predictive or prognostic potential in NSCLC.

In this study, we investigated the relationship between pretreatment peripheral blood cell parameters and circulating cytokines and the objective response rate (ORR), onset of irAEs, progression-free survival (PFS), and overall survival (OS) in patients with advanced NSCLC. We compared the differences between patients receiving immunotherapy only and patients who received chemoimmunotherapy. Moreover, changes during course of disease were correlated with the respective outcome variables. We present the following article in accordance with the REMARK reporting checklist (available at https:// dx.doi.org/10.21037/tlcr-21-710).

\section{Methods}

\section{Patients}

A total of 103 patients, aged 18 years or older, with pathologically confirmed stage III/IV advanced NSCLC, who received at least 2 doses of anti-PD-(L)1 monotherapy, or anti-PD-(L)1-based combination therapy at Peking Union Medical College Hospital (PUMCH) from January 
2015 to December 2020, were retrospectively enrolled in this study. The pretreatment routine blood test results and at least 1 type of serum cytokine result were available for all patients. The pretreatment blood results were examined within 7 days prior to immunotherapy initiation. Of the 103 patients, on-treatment peripheral blood results that were examined 6 to 8 weeks after immunotherapy initiation, were available for 59 patients (see Figure S1). ICIs were administered intravenously every 21 days according to label. The follow-up period ended on December 31, 2020.

\section{Data collection}

The clinicopathologic features and treatment history of the patients were reviewed. The peripheral blood test results retrieved included the absolute neutrophil count (ANC), absolute eosinophil count (AEC), absolute lymphocyte count (ALC), serum lactate dehydrogenase (LDH), serum hypersensitive C-reactive protein (hsCRP), erythrocyte sedimentation rate (ESR), serum IL-6 level, serum IL-8 level, serum IL-10 level, serum tumor necrosis factor- $\alpha$ (TNF- $\alpha$ level, and lymphocyte subset parameters, including $\mathrm{B}$ cell (cluster of differentiation $\mathrm{CD}^{-} \mathrm{CD} 19^{+}$lymphocytes) count, natural killer (NK) cell $\left(\mathrm{CD}^{-} \mathrm{CD} 16^{+} \mathrm{CD} 56^{+}\right.$ lymphocytes) count, $\mathrm{CD} 4^{+} \mathrm{T}$ lymphocyte count, and $\mathrm{CD} 8^{+} \mathrm{T}$ lymphocyte count. Cytokines were examined by electrochemiluminescence immunoassay. Complex parameters were calculated as follows: (I) the NLR was calculated by dividing the ANC by the ALC; (II) the PLR was calculated by dividing the platelet count by the ALC; and (III) the PNI was calculated as $10 \times$ albumin value $(\mathrm{g} / \mathrm{dL})$ $+0.005 \times$ ALC. The following cutoff values were set: (I) a NLR greater than 5 (29); and (II) a PNI greater than 45 (18).

In relation to the on-treatment peripheral blood parameters, including the ALC, AEC, NLR, PLR, B cell count, NK cell count, $\mathrm{CD}^{+} \mathrm{T}$ lymphocyte count, $\mathrm{CD} 8^{+}$ T lymphocyte count, LDH level, ESR, and hsCRP level, we evaluated the relationship between the interquartile range (IQR) of changes between the pretreatment and on-treatment values. For cytokines, including IL-6, IL-8, IL-10, and TNF $\alpha$, an increase or decrease of more than $40 \%$ in the pretreatment value was considered to represent significant biological variation (28).

This study was performed in accordance with the principles for Good Clinical Practice and the Declaration of Helsinki (as revised in 2013). The study was approved by the Internal Review Board of PUMCH on August 31, 2020 (protocol number SK-135) and individual consent for this retrospective analysis was waived.

\section{Response assessments and recognition of irAEs}

Response assessment was performed according to the Response Evaluation Criteria in Solid Tumors version 1.1 (RECIST 1.1) by computed tomography scans every 6 to 8 weeks after the administration of the first dose of immunotherapy (30). Best overall response (BOR) refers to best percentage change from baseline in investigatorassessed tumor size during ICIs or chemoimmunotherapy for patients with measurable lesions. IrAEs were defined by physicians after alternative diagnoses had been excluded based on (I) pathologic evidence of irAEs; (II) multidisciplinary adjudication involving 2 or more oncologists; (III) clinical improvement with irAE-based treatment. The National Cancer Institute Common Terminology Criteria for Adverse Events (CTCAE; version 4.0) was used to classify adverse events. PFS was defined as the time from the start of immunotherapy to the date of clinician-assessed radiographic progression, death, or last follow-up appointment, whichever occurred first. OS was defined as the date of the first dose of immunotherapy until death from any cause or the last follow-up appointment.

\section{Statistical analysis}

Patient characteristics are presented as descriptive statistics. Continuous variables are expressed as the median IQR, and categorical variables are expressed as numbers (\%). Student's $\mathrm{t}$ and Kruskal-Wallis $\mathrm{H}$ tests were used to compare continuous variables. Chi-squared test was used to compare categorical variables. A logistic regression analysis was conducted to explore correlations between peripheral blood markers and the best overall response (BOR) and the onset of irAEs. A Kaplan-Meier analysis of PFS and OS was performed based on the cutoff values or IQRs, and differences between each group were assessed with the log rank test. The hazard ratios (HRs) and 95\% confidence intervals (CI) were calculated with the multivariate Cox proportional hazard model. For all analyses, differences with a 2 -tailed $\mathrm{P}<0.05$ were considered statistically significant. All statistical analyses were performed using the Statistical Analysis System Version 9.2 (SAS Institute, Cary, NC, USA). The figures were developed in GraphPad Prism (San Diego, CA, USA). 
Table 1 Patients' clinical characteristics at baseline $(\mathrm{n}=103)$

\begin{tabular}{|c|c|}
\hline Characteristic & N (\%) \\
\hline Age (yrs) at ICI initiation, median (IQR) & $66(61,71)$ \\
\hline \multicolumn{2}{|l|}{ Gender } \\
\hline Male & $68(66.0)$ \\
\hline Female & $35(34.0)$ \\
\hline BMI before ICI treatment, median (IQR) & $22.3(20.3,24.1)$ \\
\hline \multicolumn{2}{|l|}{ ECOG PS } \\
\hline $0-1$ & $97(94.2)$ \\
\hline$>1$ & $6(5.8)$ \\
\hline \multicolumn{2}{|l|}{ Smoking status } \\
\hline Never & $45(43.7)$ \\
\hline Current & $36(35.0)$ \\
\hline Former & $22(21.3)$ \\
\hline \multicolumn{2}{|l|}{ Histology } \\
\hline Non-squamous & $66(64.1)$ \\
\hline Squamous & $37(35.9)$ \\
\hline \multicolumn{2}{|l|}{ Driving mutation status } \\
\hline Positive & $38(36.9)$ \\
\hline EGFR & $22(21.4)$ \\
\hline ALK & $1(1.0)$ \\
\hline KRAS & $9(8.7)$ \\
\hline Other & $6(5.8)$ \\
\hline Negative & $65(63.1)$ \\
\hline \multicolumn{2}{|l|}{ PD-L1 expression } \\
\hline Negative & $22(21.4)$ \\
\hline $0-50 \%$ & $13(12.6)$ \\
\hline$\geq 50 \%$ & $15(14.6)$ \\
\hline Not available & $53(51.4)$ \\
\hline \multicolumn{2}{|l|}{ Stage at diagnosis } \\
\hline III & $20(19.4)$ \\
\hline IV & $83(80.6)$ \\
\hline \multicolumn{2}{|l|}{ Number of metastatic sites } \\
\hline$<3$ & $80(77.7)$ \\
\hline$\geq 3$ & $23(22.3)$ \\
\hline
\end{tabular}

Table 1 (continued)
Table 1 (continued)

\begin{tabular}{|c|c|}
\hline Characteristic & N (\%) \\
\hline \multicolumn{2}{|l|}{ Line of therapy } \\
\hline $1 \mathrm{st}$ & $62(60.2)$ \\
\hline 2nd & $24(23.3)$ \\
\hline 3rd or more & $17(16.5)$ \\
\hline \multicolumn{2}{|l|}{ Treatment received } \\
\hline Immunotherapy & $32(31.1)$ \\
\hline Chemoimmunotherapy & 71 (68.9) \\
\hline \multicolumn{2}{|c|}{$\begin{array}{l}\text { ICI, immune checkpoint inhibitors; IQR, interquartile range; BMI, } \\
\text { body mass index; EGFR, epidermal growth factor receptor; } \\
\text { ALK, anaplastic Iymphoma kinase; KRAS, kirsten rat sarcoma } \\
\text { viral oncogene; PD-L1, programmed death-ligand 1; ECOG PS, } \\
\text { Eastern Cooperative Oncology Group performance status. }\end{array}$} \\
\hline
\end{tabular}

\section{Results}

\section{Patient characteristics, treatment, and efficacy}

Pretreatment routine blood test results and clinicopathological data were available for all 103 patients. Among these patients, the following specific parameters were available: IL-6 for 103 patients (100\%), the PNI for 101 patients (98.1\%), lymphocyte subsets for 96 patients $(93.2 \%)$, IL-8 for 91 patients (88.3\%), IL-10 for 91 patients (88.3\%), TNF- $\alpha$ for 77 patients (74.8\%), LDH for 73 patients (70.9\%), hsCRP for 62 patients (60.2\%), and ESR for 55 patients (53.4\%). Patient's clinical characteristics are summarized in Table 1. Of the 103 patients, $64.1 \%(\mathrm{n}=66)$ were diagnosed with non-squamous NSCLC, while 35.9\% (n=37) had a squamous NSCLC, 94.2\% ( $\mathrm{n}=97)$ had an Eastern Cooperative Oncology Group (ECOG) performance status (PS) of $0-1$, and $80.6 \%(n=83)$ of the whole cohort were diagnosed within a metastatic disease. In terms of treatment, $31.1 \%(\mathrm{n}=32)$ received ICIs monotherapy, and $68.9 \%(\mathrm{n}=71)$ received chemoimmunotherapy, 62 patients $(60.2 \%)$ received ICI treatment in the first-line setting.

The median follow-up time was 12.9 (95\% CI: 11.4 to 14.3) months. The median PFS was 12.8 (95\% CI: 6.1 to 19.6) months, and the median OS was 23.8 (95\% CI: 13.4 to 34.2$)$ months in the whole cohort. The ORR was $43.7 \%$ [45/103, 0 complete response (CR), 45 partial response (PR)]. The disease control rate (DCR) was 91.3\% [94/103, 
0 CR, $45 \mathrm{PR}, 49$ stable disease (SD)]. In patients receiving ICIs only, the ORR was $34.4 \%(11 / 32,11 \mathrm{PR})$ while for patients receiving chemoimmunotherapy, the ORR was $45.1 \%$ (32/71, 32 PR). Patients who received first-line chemoimmunotherapy showed better ORR than patients treated with ICI in subsequent lines: $58.5 \%(31 / 53,31 \mathrm{PR})$ vs. $16.7 \%(3 / 18,3 \mathrm{PR})$.

\section{Baseline peripheral blood parameters related to the BOR}

Patient stratification into three subgroups according to BOR [CR/PR; SD; progressive disease (PD)], showed that these sub-cohorts were characterized by different pretreatment NLR $(\mathrm{P}=0.043)$, and TNF- $\alpha$ levels $(\mathrm{P}=0.013)$ (Table S1).

In multivariate analysis, peripheral blood parameters failed to remain independent prognosticators (Table S2). In patients receiving immunotherapy only, there was a significant difference in ESR among the different response groups (the median ESR value was 25 in the CR/ PR group, 24 in the SD group, and 84 in the PD group, $\mathrm{P}=0.029$, Table $\mathrm{S} 3$ ); however, no significant difference was observed in the multivariate analysis. In patients receiving chemoimmunotherapy, there was a significant difference in TNF- $\alpha$ levels among the CR/PR, SD, and PD groups (the median TNF- $\alpha$ value was 9 in the CR/PR group, 8 in the SD group, and 7.45 in the PD group, $\mathrm{P}=0.009$, Table S4). However, no significant difference was found in the logistic regression. No significant difference was observed in patients receiving first-line chemoimmunotherapy $(n=53$, Table S5).

\section{IrAEs}

A total of $36.9 \%(38 / 103)$ patients experienced 57 irAEs. Twenty-three patients developed single-system irAEs, and 15 patients developed multiple-system irAEs across a maximum of four organ systems. The most observed irAEs were rash (22.8\%), hepatic toxicity (14.0\%), and hypothyroidism (12.3\%). The most common severe irAEs (grade $\geq 3$ ) was myocarditis $(n=2)$. The detailed toxicity spectrum is provided in Table 2. Patients experiencing irAEs showed a longer PFS compared to patients without irAEs (498 vs. 269 days; Figure 1A; log rank $\mathrm{P}=0.124$ ). Similarly, the median OS of irAE patients was better compared to patients without irAEs (1,198 vs. 484 days, Figure 1B, log $\operatorname{rank} \mathrm{P}=0.071$ ).

\section{Baseline peripheral blood parameters and the incidence of irAEs}

Patients with irAEs had higher baseline ALC (1.320 vs. 1.280, $\mathrm{P}=0.021)$, lower NLR (2.915 vs. 3.990, $\mathrm{P}=0.007$ ) and lower PLR (159.560 vs. 207.780, $\mathrm{P}=0.032$ ) compared to those without irAEs (Table 3). The univariate analysis revealed differences in the ALC (OR, 2.280, 95\% CI: 1.089 to 4.775; $\mathrm{P}=0.029$ ), NLR (OR, $0.823,95 \%$ CI: 0.695 to 0.975; $\mathrm{P}=0.024)$, and PLR (OR, 0.996, 95\% CI: 0.992 to 1.000; $\mathrm{P}=0.039)$. The multivariate analysis revealed that the ALC (OR, 2.165, 95\% CI: 1.040 to $4.509 ; \mathrm{P}=0.039)$ and the number of metastatic sites (OR, 0.196, 95\% CI: 0.051 to $0.753 ; \mathrm{P}=0.018$ ) were independent risk factors for the occurrence of irAEs (Table 4).

In patients receiving immunotherapy only, the median pretreatment ALC was higher in the irAEs group compared to the non-irAEs group $(\mathrm{P}=0.038$; Table 5). The univariate analysis also revealed a positive relationship between patients' pretreatment ALC and the incidence risk of irAEs (OR, 6.461, 95\% CI: 1.067 to 39.112; $\mathrm{P}=0.042$; Table 6). In the subgroups of patients receiving chemoimmunotherapy or patients receiving chemoimmunotherapy in the first line setting, no statistically significant associations were observed (Table S6; Table S7).

\section{Relationship between pretreatment peripheral biomarkers and survival}

Patients with high PNI (>45) tended to have superior PFS and OS than those with low PNI $(\log \operatorname{rank} \mathrm{P}=0.009$ and $\log$ rank $\mathrm{P}<0.001$, respectively; Figure $2 A, 2 B)$. Additionally, patients with the highest (fourth quartile) IL-6 (>13.80 pg/mL) showed shorter PFS and OS compared to those with IL-6 in the other 3 quartiles $(\log \operatorname{rank} \mathrm{P}=0.007$ and $\mathrm{P}=0.003$, respectively; Figure 3A,3B). Moreover, a high NLR ( $>5$; log rank $\mathrm{P}=0.013$ ) and a high PLR (>median of 196.32 ; log rank $\mathrm{P}=0.025$ ) were associated with inferior OS (Figure S2A,S2B). Thus, we set the lower limit of the fourth quartile of IL-6 $(13.80 \mathrm{pg} / \mathrm{mL})$ in the whole population as the cutoff value and performed further multivariate analysis. It revealed that IL-6 $>13.80 \mathrm{pg} / \mathrm{mL}$ had a significant association with worse PFS (HR, 1.903, 95\% CI: 1.055 to 3.433; $\mathrm{P}=0.032$ ), whereas a PNI $>45$ had a significant association with better PFS (HR, 0.405, 95\% CI: 0.184 to $0.892, \mathrm{P}=0.025$ ) and $\mathrm{OS}$ (HR, 0.294, 95\% CI: 0.123 to $0.703 ; \mathrm{P}=0.006$; see Table 7).

In the subset of patients receiving immunotherapy only, 
Table 2 Distribution of immune-related adverse events

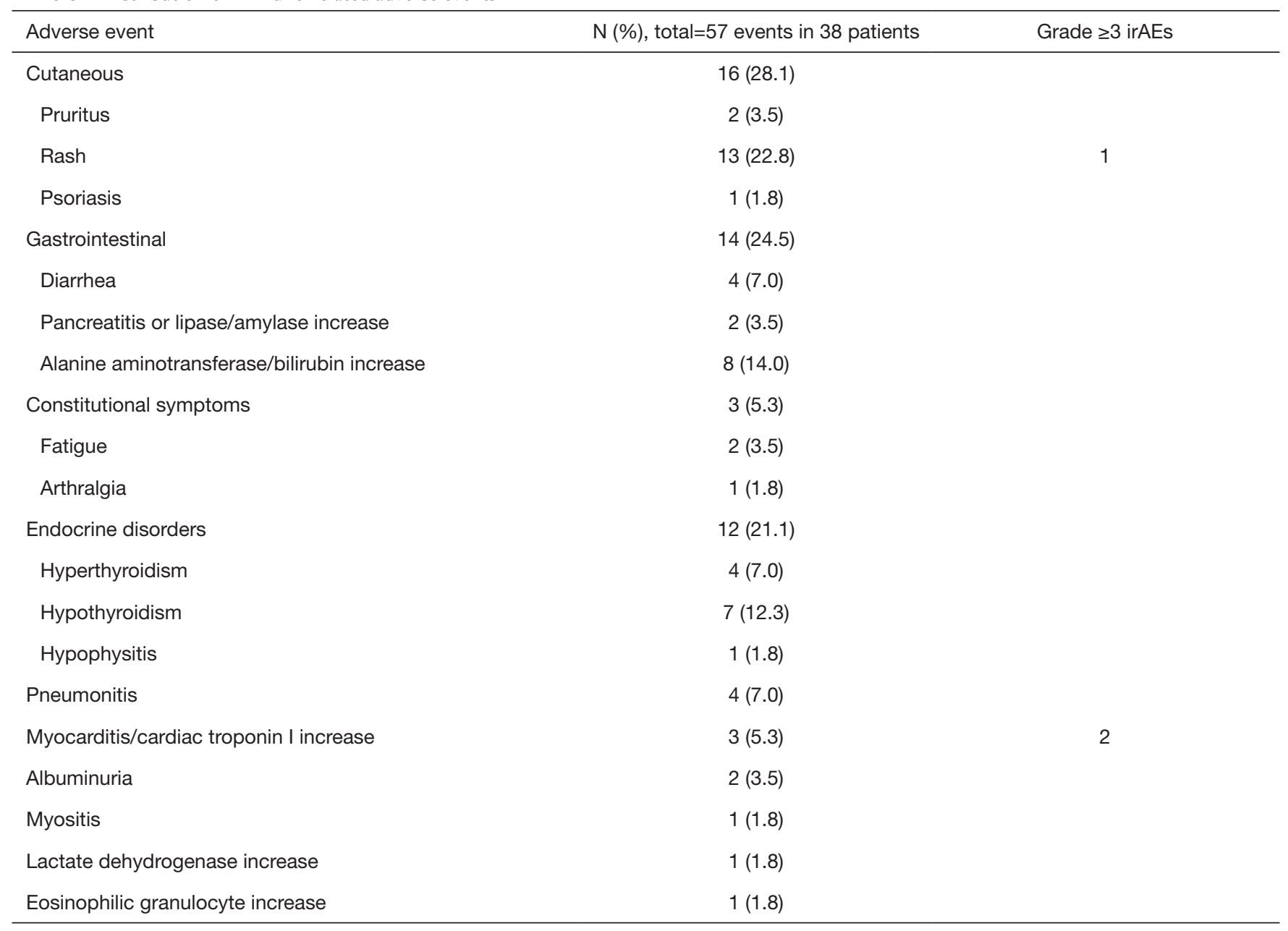

irAEs, immune-related adverse events.
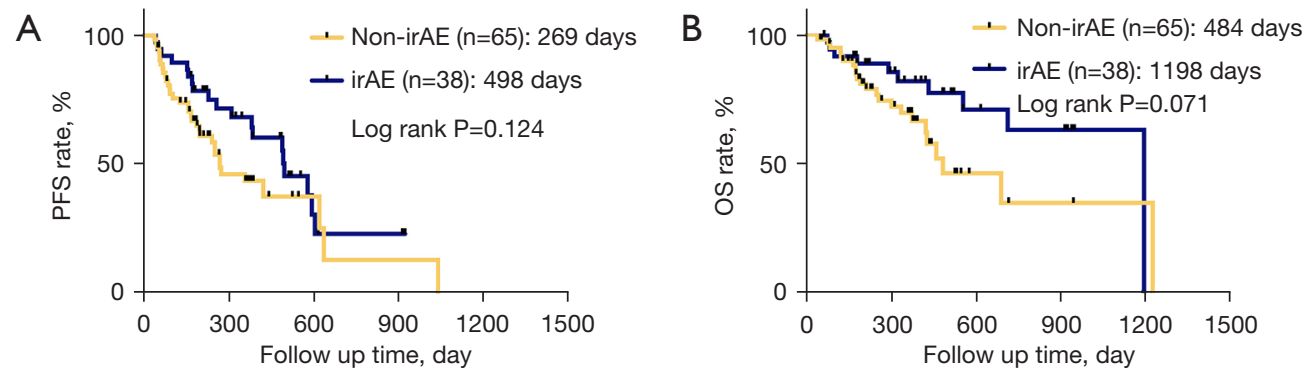

No. at risk

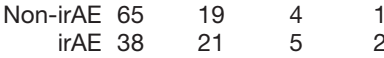

No. at risk

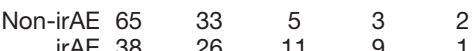

Figure 1 Kaplan-Meier analysis of PFS (A) and OS (B) for all patients ( $\mathrm{n}=103)$ with ICI-treated advanced NSCLC according to the incidence of irAEs. The P values were calculated with the log rank test. PFS, progression-free-survival; OS, overall survival; ICI, immune checkpoint inhibitor; NSCLC, non-small cell lung cancer; irAEs, immune-related adverse events. 
Table 3 Clinical-pathological characteristics and baseline peripheral blood parameters' and the risk irAEs

\begin{tabular}{|c|c|c|c|}
\hline Clinical or biological marker & Without irAEs $(n=65)$ & With irAEs $(n=38)$ & $P$ value \\
\hline BMI $\left(\mathrm{kg} / \mathrm{m}^{2}\right)$ & $22.00(20.30,24.10)$ & $22.30(20.80,23.90)$ & 0.486 \\
\hline \multicolumn{4}{|l|}{ Blood routine test } \\
\hline ANC & $5.37(3.98,7.08)$ & $4.81(2.94,5.74)$ & 0.246 \\
\hline NLR & $3.99(2.81,6.89)$ & $2.92(2.33,4.76)$ & 0.007 \\
\hline$N L R<5$ & $40(61.5 \%)$ & $29(76.3 \%)$ & 0.124 \\
\hline$N L R \geq 5$ & $25(38.5 \%)$ & $9(23.7 \%)$ & \\
\hline AEC & $0.13(0.04,0.23)$ & $0.15(0.06,0.29)$ & 0.071 \\
\hline Lymphocyte subsets test & $\mathrm{N}=59$ & $\mathrm{~N}=37$ & \\
\hline B cell & $98.00(66.00,157.00)$ & $107.00(77.00,183.00)$ & 0.361 \\
\hline NK cell & $247.00(156.00,419.00)$ & $271.00(194.00,371.00)$ & 0.978 \\
\hline $\mathrm{CD4}^{+} \mathrm{T}$ lymphocyte & $446.00(263.00,622.00)$ & $542.00(369.00,703.00)$ & 0.091 \\
\hline $\mathrm{CD8}^{+} \mathrm{T}$ lymphocyte & $338.00(195.00,465.00)$ & $338.00(285.00,428.00)$ & 0.219 \\
\hline $\mathrm{LDH}$ & $\begin{array}{c}\mathrm{N}=46 \\
221.00(188.00,254.00)\end{array}$ & $\begin{array}{c}\mathrm{N}=27 \\
216.00(177.00,249.00)\end{array}$ & 0.527 \\
\hline IL-8 & $\begin{array}{c}\mathrm{N}=63 \\
25.00(17.00,57.00)\end{array}$ & $\begin{array}{c}\mathrm{N}=28 \\
45.00(16.00,90.00)\end{array}$ & 0.256 \\
\hline IL-10 & $\begin{array}{c}\mathrm{N}=63 \\
5.00(5.00,5.00)\end{array}$ & $\begin{array}{c}\mathrm{N}=28 \\
5.00(5.00,5.00)\end{array}$ & 0.351 \\
\hline $\mathrm{TNF} \alpha$ & $\begin{array}{c}N=56 \\
8.10(5.50,10.50)\end{array}$ & $\begin{array}{c}\mathrm{N}=21 \\
7.90(6.20,9.30)\end{array}$ & 0.130 \\
\hline ECOG PS & & & 0.852 \\
\hline $0-1$ & $61(93.8 \%)$ & $36(94.7 \%)$ & \\
\hline$\geq 1$ & $4(6.2 \%)$ & $2(5.3 \%)$ & \\
\hline Number of metastases & & & 0.007 \\
\hline$<3$ & $45(69.2 \%)$ & $35(92.1 \%)$ & \\
\hline$\geq 3$ & 20 (30.8\%) & $3(7.9 \%)$ & \\
\hline
\end{tabular}

Table 3 (continued) 
Table 3 (continued)

\begin{tabular}{lcc}
\hline Clinical or biological marker & Without irAEs $(n=65)$ & With irAEs $(n=38)$ \\
\hline ICl treatment type & $65(100.0 \%)$ & $34(89.5 \%)$ \\
Anti-PD-(L)-1 & 0 & $4(10.5 \%)$ \\
Anti-PD-(L)1 \& anti-CTLA4 & & 0.017 \\
Line of therapy & $38(56.9 \%)$ & $25(65.8 \%)$ \\
1st & $14(21.5 \%)$ & $10(26.3 \%)$ \\
2nd & $14(21.5 \%)$ & $3(7.9 \%)$ \\
3rd or more & & 0.197 \\
Treatment received & $18(27.7 \%)$ & $14(36.8 \%)$ \\
Immunotherapy & $47(72.3 \%)$ & $24(63.2 \%)$ \\
Chemoimmunotherapy & 0.333 \\
\hline
\end{tabular}

irAEs, immune-related adverse events; BMI, body mass index; ANC, absolute neutrophil count; ALC, absolute lymphocyte count; NLR, neutrophil-lymphocyte ratio; AEC, absolute eosinophils count; PLR, platelet-lymphocyte ratio; PNI, prognostic nutrition index; LDH, lactate dehydrogenase; hsCRP, hypersensitive C-reactive protein; ESR, erythrocyte sedimentation rate; IL-6, interleukin-6; IL-8, interleukin-8; TNF- $\alpha$, tumor necrosis factor- $\alpha$; ECOG PS, Eastern Cooperative Oncology Group performance status; ICl, immune checkpoint inhibitor; PD-(L)-1, programmed death-(ligand)-1; CTLA4, cytotoxic T-lymphocyte-associated protein 4.

Table 4 Univariate and multivariate analyses of risk factors for irAEs ( $\mathrm{n}=103)$

\begin{tabular}{|c|c|c|c|c|c|c|c|}
\hline Variable & Category & \multicolumn{2}{|c|}{ Univariate } & \multicolumn{4}{|c|}{ Multivariate } \\
\hline ALC & - & 2.280 & $1.089-4.775$ & 0.029 & 2.165 & $1.040-4.509$ & 0.039 \\
\hline NLR & - & 0.823 & $0.695-0.975$ & 0.024 & & & NS \\
\hline AEC & - & 3.185 & $0.970-10.454$ & 0.056 & & & \\
\hline $\mathrm{PNI}$ & $>45$ & 1.740 & $0.758-3.995$ & 0.191 & & & \\
\hline TNF- $\alpha$ & - & 0.958 & $0.868-1.056$ & 0.385 & & & \\
\hline IL-6 & - & 0.993 & $0.964-1.023$ & 0.638 & & & \\
\hline IL-8 & - & 1.002 & $0.999-1.005$ & 0.184 & & & \\
\hline
\end{tabular}

irAEs, immune-related adverse events; ALC, absolute lymphocyte count; NLR, neutrophil-lymphocyte ratio; NS, not significant; AEC, absolute eosinophils count; PLR, platelet-lymphocyte ratio; PNI, prognostic nutrition index; IL-6, interleukin-6; IL-8, interleukin-8; ICI, immune checkpoint inhibitors.

high NLR and low PNI were associated with poor survival compared to the low NLR group and the high PNI group in terms of both: PFS (log rank $\mathrm{P}<0.001$, and $\log$ rank $\mathrm{P}=0.006$, respectively) and $\mathrm{OS}(\log$ rank $\mathrm{P}=0.011$, and $\log$ rank $\mathrm{P}=0.019$, respectively; Figure S3A-S3D). Patients with a high PLR (>median, which was 179.94) had worse PFS ( $\log$ rank $\mathrm{P}=0.022$; Figure $\mathrm{S} 3 \mathrm{E}$ ) compared to the low PLR group. Moreover, patients with IL-8 higher than the $3^{\text {rd }}$ number of IQR, which is $76 \mathrm{pg} / \mathrm{mL}$, showed worse PFS $(\log$ rank $\mathrm{P}<0.001$, Figure S3F) compared to patients with 
Table 5 Baseline peripheral blood parameters' the incidence of irAEs in patients only receiving immunotherapy $(\mathrm{n}=32)$

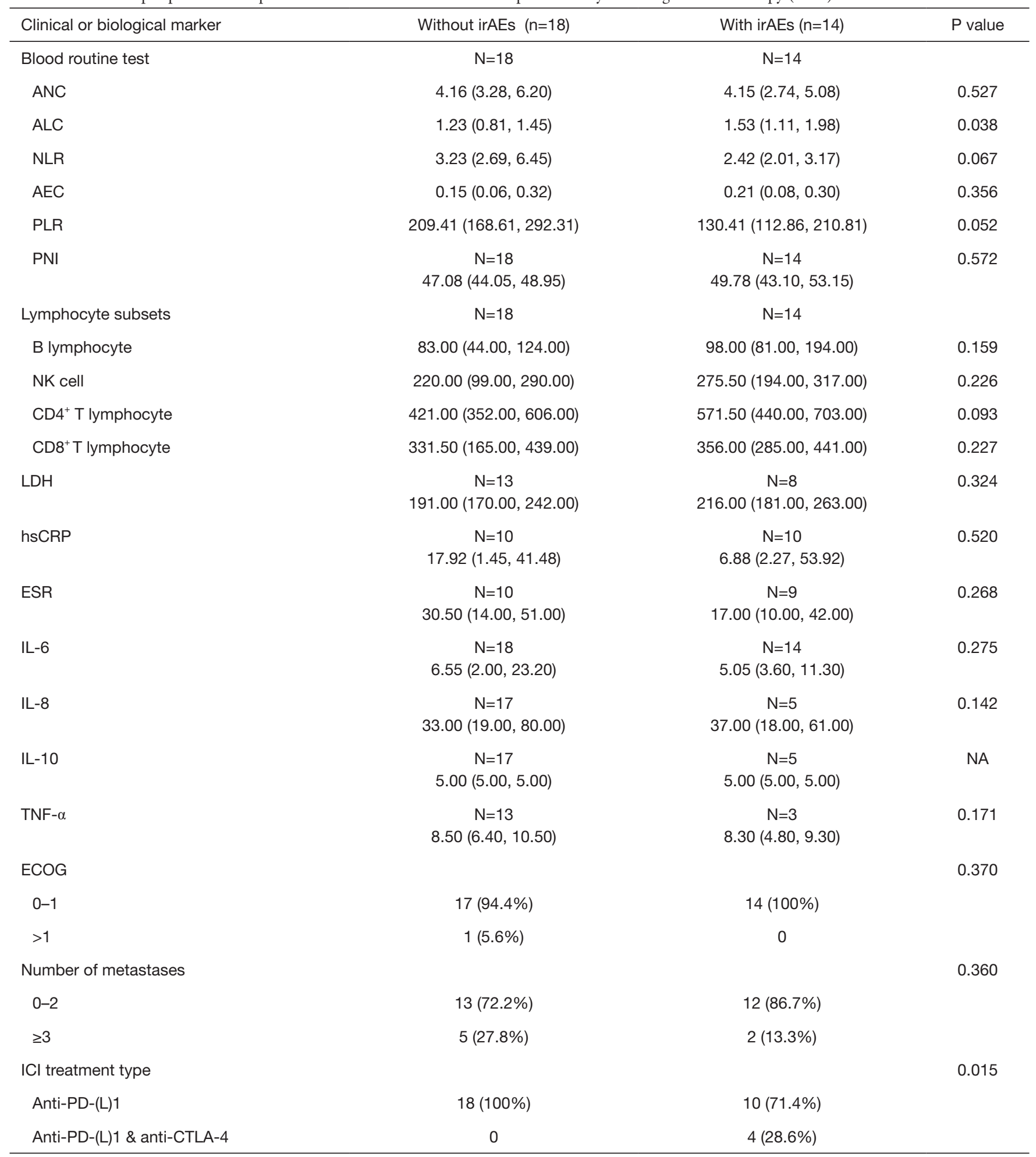

Table 4 (continued) 
Table 4 (continued)

\begin{tabular}{lcc}
\hline Clinical or biological marker & Without irAEs $(n=18)$ & With irAEs $(n=14)$ \\
\hline Line of therapy & & \\
1st & $4(22.2 \%)$ & $5(33.3 \%)$ \\
2nd & $9(50.0 \%)$ & $9(60.0 \%)$ \\
3rd or more & $5(27.8 \%)$ & 0 \\
\hline
\end{tabular}

irAEs, immune-related adverse events; ANC, absolute neutrophil count; ALC, absolute lymphocyte count; NLR, neutrophil-lymphocyte ratio; AEC, absolute eosinophils count; PLR, platelet-lymphocyte ratio; PNI, prognostic nutrition index; $\mathrm{LDH}$, lactate dehydrogenase; hsCRP, hypersensitive C-reactive protein; ESR, erythrocyte sedimentation rate; IL-6, interleukin-6; IL-8, interleukin-8; IL-10, interleukin-10; TNF- $\alpha$, tumor necrosis factor- $\alpha$; ECOG PS, Eastern Cooperative Oncology Group performance status; ICl, immune checkpoint inhibitors; PD-(L)-1, programmed death-(ligand)-1; CTLA4, cytotoxic T-lymphocyte-associated protein 4.

Table 6 Univariate analysis of risk factors for irAEs in patients receiving ICIs treatment $(\mathrm{n}=32)$

\begin{tabular}{|c|c|c|c|c|}
\hline Variable & Category & \multicolumn{3}{|c|}{ Univariate } \\
\hline ALC & - & 6.461 & $1.067-39.112$ & 0.042 \\
\hline NLR & - & 0.718 & $0.481-1.070$ & 0.104 \\
\hline AEC & - & 3.249 & $0.264-40.008$ & 0.358 \\
\hline PNI & - & 1.035 & $0.921-1.163$ & 0.560 \\
\hline TNF- $\alpha$ & - & 0.881 & $0.516-1.504$ & 0.642 \\
\hline IL6 & - & 0.977 & $0.933-1.023$ & 0.319 \\
\hline IL8 & - & 0.993 & $0.973-1.014$ & 0.498 \\
\hline
\end{tabular}

irAEs, immune-related adverse events; ALC, absolute lymphocyte count; NLR, neutrophil-lymphocyte ratio; AEC, absolute eosinophils count; PLR, platelet-lymphocyte ratio; PNI, prognostic nutrition index; TNF- $\alpha$, tumor necrosis factor- $\alpha$; IL-6, interleukin-6; IL-8, interleukin-8; ICl, immune checkpoint inhibitors.
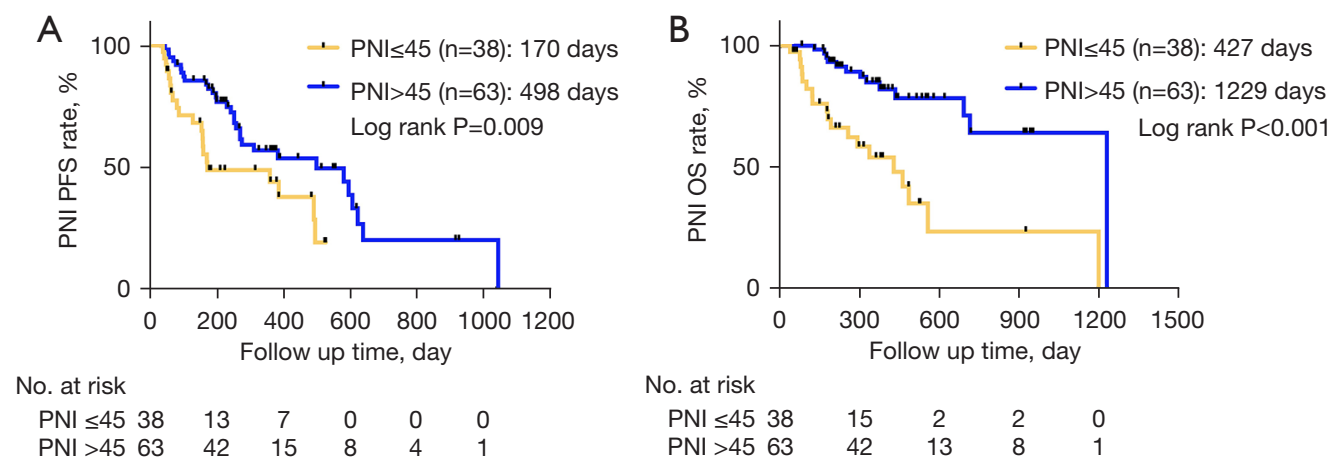

Figure 2 Kaplan-Meier analysis of PFS (A) and OS (B) for all patients according to the pretreatment PNI ( $\leq 45 v s$. $>45)$ (n=103, PNI was available in 101 patients). The $\mathrm{P}$ values were calculated with the log rank test. PFS, progression free survival; OS, overall survival; PNI, prognostic nutritional index. 
A

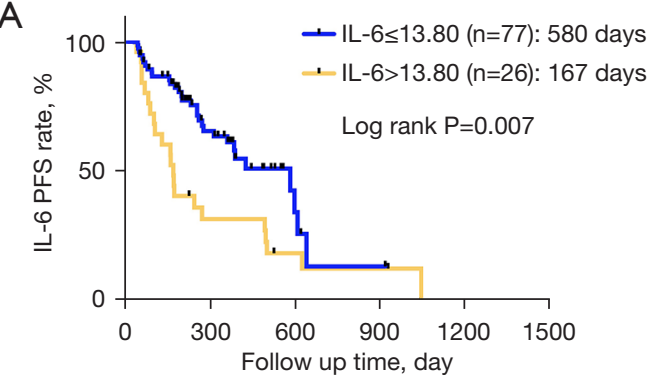

No. at risk

Q1-Q3 $77 \quad 33 \quad 5 \quad 2$

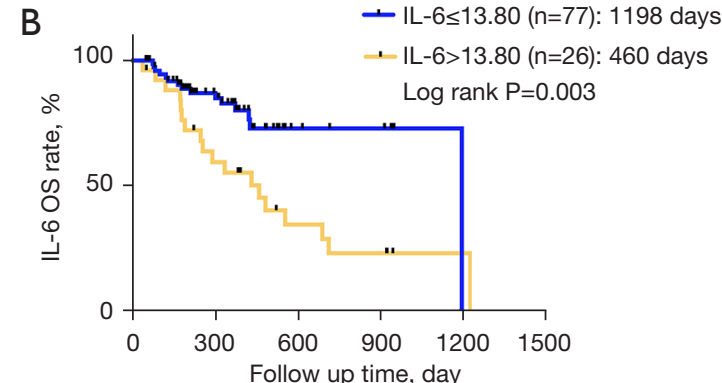

No. at risk

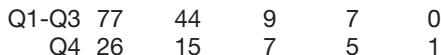

Figure 3 Kaplan-Meier analysis of PFS (A) and OS (B) for all patients (n=103) according to pretreatment IL-6 level ( $\leq 13.80$ vs. $>13.80 \mathrm{pg} / \mathrm{mL})$. The $\mathrm{P}$ values were calculated with the log rank test. PFS, progression free survival; OS, overall survival; IL-6, interleukin-6.

Table 7 Multivariate analyses of PFS and OS for all patients ( $\mathrm{n}=103$, with 101 patients' data available)

\begin{tabular}{|c|c|c|c|c|c|c|c|}
\hline Variable & Category & \multicolumn{3}{|c|}{ PFS } & \multicolumn{3}{|c|}{ OS } \\
\hline NLR & $>5$ & 1.071 & $0.457-2.508$ & 0.874 & 1.133 & $0.386-3.330$ & 0.820 \\
\hline PLR & $>196.52$ & 0.603 & $0.258-1.412$ & 0.244 & 0.877 & $0.297-2.594$ & 0.813 \\
\hline PNI & $>45$ & 0.405 & $0.184-0.892$ & 0.025 & 0.294 & $0.123-0.703$ & 0.006 \\
\hline
\end{tabular}

PFS, progression-free survival; OS, overall survival; HR, hazard ratio; CI, confidence interval; PLR, platelet-to-lymphocyte ratio; NLR, neutrophil-to-lymphocyte ratio; PNI, prognostic nutritional index; IL-6, interleukin-6.

low IL-8. IL-6 $=11.30 \mathrm{pg} / \mathrm{mL}$ was a meaningful cutoff of PFS (log rank $\mathrm{P}=0.028)$ and $\mathrm{OS}(\log \operatorname{rank} \mathrm{P}=0.038)$ for patients receiving immunotherapy only (Figure 4). In the multivariate Cox regression yielded a correlation between high PNI was associated with better OS (HR 0.068, 95\% CI: 0.005 to $0.876 ; \mathrm{P}=0.039$; Table 8 ).

In the subset of patients receiving chemoimmunotherapy, with IL-6 $>11.85 \mathrm{pg} / \mathrm{mL}\left({ }^{\text {rd }}\right.$ number of IQR) was associated with inferior PFS ( $\log$ rank $\mathrm{P}=0.015$, Figure $5 A$ ) and $\mathrm{OS}(\log$ rank $\mathrm{P}=0.047$, Figure $5 B$ ) compared to patients with IL-6 $\leq 11.85 \mathrm{pg} / \mathrm{mL}$. Patients with PNI $>45$ showed improved OS ( $\log$ rank $\mathrm{P}=0.001$; Figure S4A). Conversely, patients with high PLR (>median, which was 200) had worse OS (log rank $\mathrm{P}=0.044$, Figure $\mathrm{S} 4 \mathrm{~B})$. In multivariate Cox regression analysis, high PLR (HR 3.594, 95\% CI: 1.096 to 11.789 , $\mathrm{P}=0.035$ ), low PNI (HR 0.273, 95\% CI: 0.082 to 0.907 , $\mathrm{P}=0.034$ ) and high IL-6 (HR 2.195, 95\% CI: 1.008 to 4.779, $\mathrm{P}=0.048$ ) remained independent poor prognostic factors for PFS. In multivariate analysis for OS including the same covariates, no independent risk factor was detected
(Table 9). In patients receiving chemoimmunotherapy as first-line treatment, high PLR $(>214.4, \log$ rank $\mathrm{P}=0.009$, Figure S5A) and low PNI $(\leq 45, \log$ rank $\mathrm{P}=0.047$, Figure $\mathrm{S} 5 \mathrm{~B})$ were associated with worse OS. In patients with high IL-6 (>11.30 pg/mL, $3^{\text {rd }}$ number of IQR), there was a trend towards poor OS $(\log \operatorname{rank} \mathrm{P}=0.056$, Figure S5C). The multivariate analysis failed to reveal any independent risk factors in this subgroup of patients (Table S8).

\section{Changes between pretreatment and on-treatment peripheral blood parameters}

Pretreatment and on-treatment blood results were available for 59 patients. Of those, 13 received ICIs and 46 received chemoimmunotherapy. Routine blood and IL-6 results were available for all 59 patients. Other parameters were available as follows: 57 patients had PNI results, 55 patients had IL-8 results, 46 patients had lymphocyte subset results, 45 patients had TNF- $\alpha$ results, 37 patients had LDH results, 27 patients had had CRP results, and 26 patients 

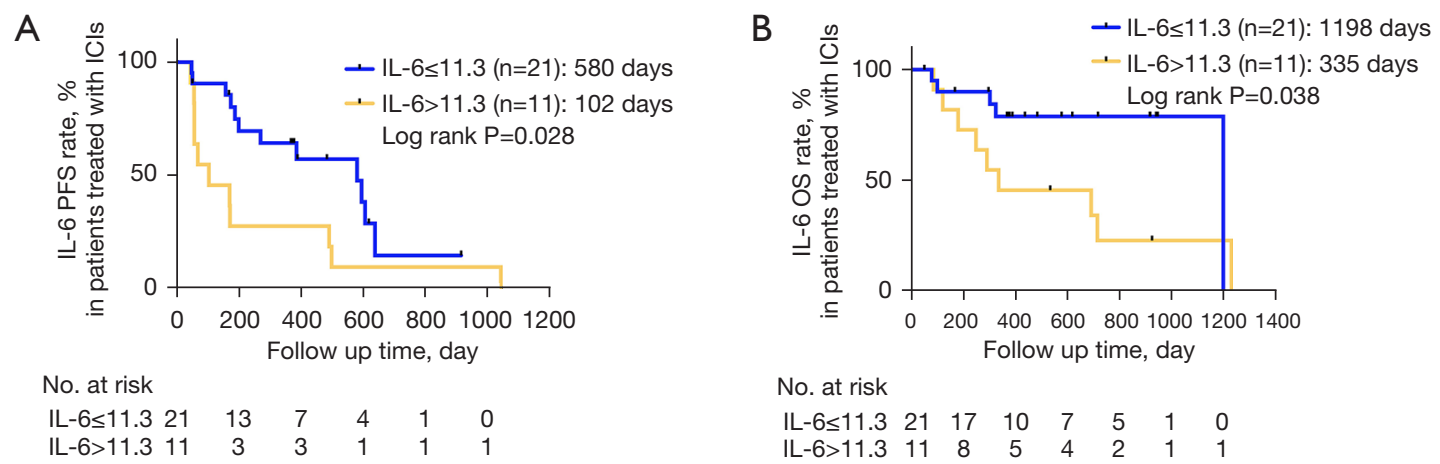

Figure 4 Kaplan-Meier analysis of PFS (A) and OS (B) for patients receiving immunotherapy only (n=32) according to pretreatment level of IL-6 ( $\leq 11.30$ vs. $>11.30 \mathrm{pg} / \mathrm{mL}$ ). The P values were calculated with the log-rank test. PFS, progression free survival; OS, overall survival; IL6 , interleukin-6.

Table 8 Multivariate analyses of PFS and OS for patients receiving immunotherapy alone ( $\mathrm{n}=32$, with 21 patient's data available)

\begin{tabular}{|c|c|c|c|c|c|c|c|}
\hline Variable & Category & \multicolumn{3}{|c|}{ PFS } & \multicolumn{3}{|c|}{ OS } \\
\hline NLR & $>5$ & 1.524 & $0.194-11.970$ & 0.689 & 67933.508 & $0.000-9.517 E+126$ & 0.938 \\
\hline PLR & $>179.94$ & 2.089 & $0.269-16.198$ & 0.481 & 0.000 & $0.000-1.310 E+117$ & 0.936 \\
\hline PNI & $>45$ & 1.040 & $0.223-4.845$ & 0.960 & 0.068 & $0.005-0.876$ & 0.039 \\
\hline IL-8 & $>76$ & 4.454 & $0.598-33.154$ & 0.145 & 4.227 & $0.483-36.991$ & 0.193 \\
\hline
\end{tabular}

PFS, progression-free survival; OS, overall survival; HR, hazard ratio; Cl, confidence interval; PLR, platelet-to-lymphocyte ratio; NLR, neutrophil-to-lymphocyte ratio; PNI, prognostic nutritional index; IL-6, interleukin-6; IL-8, interleukin-8.
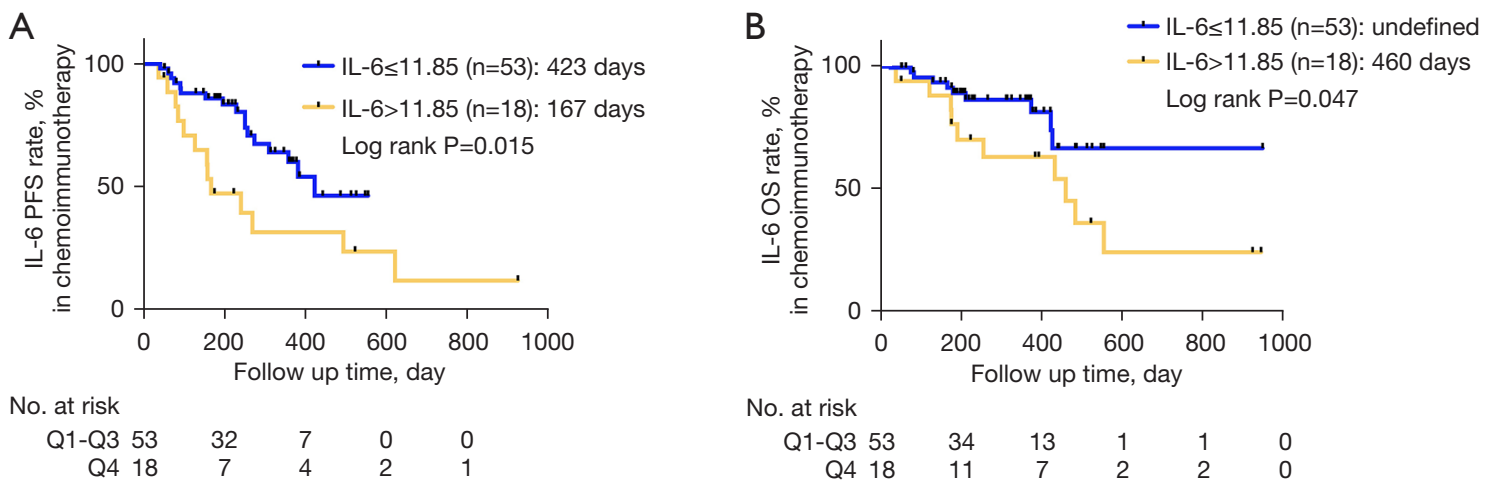

Figure 5 Kaplan-Meier analysis of PFS (A) and OS (B) for patients receiving chemoimmunotherapy (n=71) according to pretreatment level of IL-6 ( $\leq 11.85$ vs. $>11.85 \mathrm{pg} / \mathrm{mL})$. The P values were calculated with the log rank test. PFS, progression free survival; OS, overall survival; IL-6, interleukin-6. 
Table 9 Multivariate analyses of PFS and OS for patients receiving chemoimmunotherapy ( $\mathrm{n}=73$, with 71 patient's data available)

\begin{tabular}{|c|c|c|c|c|c|c|c|}
\hline Variable & Category & \multicolumn{3}{|c|}{ PFS } & \multicolumn{3}{|c|}{ OS } \\
\hline PLR & $>200$ & 3.594 & $1.096-11.789$ & 0.035 & 1.223 & $0.330-4.532$ & 0.764 \\
\hline PNI & $>45$ & 0.273 & $0.082-0.907$ & 0.034 & 0.252 & $0.061-1.045$ & 0.058 \\
\hline IL-6 & $>11.85$ & 2.195 & $1.008-4.779$ & 0.048 & 1.713 & $0.614-4.780$ & 0.304 \\
\hline
\end{tabular}

PFS, progression-free survival; OS, overall survival; HR, hazard ratio; Cl, confidence interval; PLR, platelet-to-lymphocyte ratio; PNI, prognostic nutritional index; IL-6, interleukin-6.

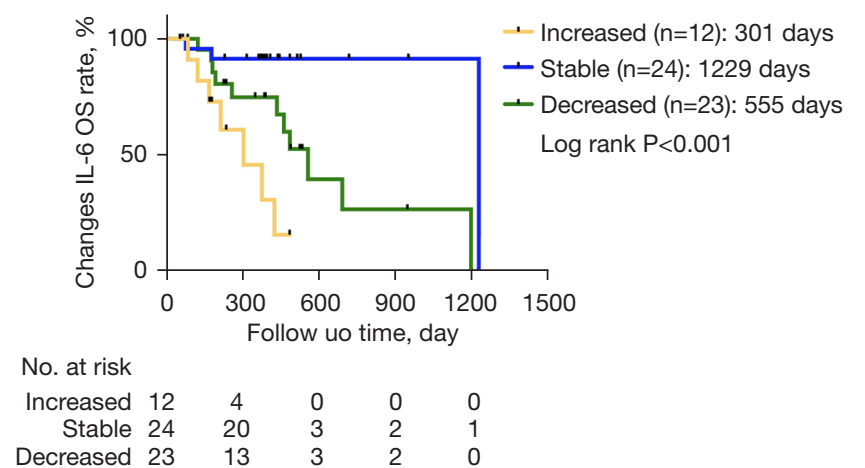

Figure 6 Kaplan-Meier analysis of OS for all patients $(n=59)$ according to changes between pretreatment and on-treatment levels of IL-6 [increased ( $>40 \%$ ), stable (decreased $\leq 40 \%$ or increased $\leq 40 \%)$, vs. decreased $(>40 \%)]$. The $\mathrm{P}$ value was calculated with the log-rank test. OS, overall survival; IL-6, interleukin-6.

had ESR results.

Analyses were conducted to examine changes between pretreatment and on-treatment peripheral blood parameters and the BOR, incidence of irAEs, and survival. There was a significant difference in the IQR changes between pretreatment and on-treatment $\mathrm{CD}^{+}{ }^{+} \mathrm{T}$ lymphocyte counts that was related to the BOR (patients with $\mathrm{Q} 2$ of $\mathrm{CD}^{+} \mathrm{T}$ lymphocyte changes more likely to be in the PD group, $\mathrm{P}=0.043$; Table S9). No parameter was found to be related to irAE incidence (Table S10). In terms of the analyses of patients receiving immunotherapy only and patients receiving chemoimmunotherapy, no significant difference was found between either BOR or irAE incidence.

Further, an increased IL-6 ( $>40 \%$ compared to pretreatment) was associated with inferior OS in the whole population ( $\log$ rank $\mathrm{P}<0.001$; Figure 6). The analysis of pretreatment and on-treatment blood parameters of patients receiving immunotherapy only and those receiving chemoimmunotherapy showed no significant differences
(Table S11).

\section{Discussion}

In this cohort, high levels of pretreatment absolute lymphocytes were associated with an increased risk for irAEs. Moreover, a higher baseline level of PNI $(>45)$ was associated with better survival, while a higher IL-6 $(>13.80 \mathrm{pg} / \mathrm{mL}$ in the whole population, $>11.30 \mathrm{pg} / \mathrm{mL}$ in the immunotherapy group, and $>11.85 \mathrm{pg} / \mathrm{mL}$ in the chemoimmunotherapy group) was associated with worse survival. Increased IL-6 levels after immunotherapy initiation were associated with worse OS in the whole population. Our study adds additional evidence on the prognostic relevance of blood-based biomarkers in NSCLC patients treated with ICIs.

Our results revealed a correlation between high pretreatment ALC and irAE incidence both in the whole population and patients receiving ICI only. These results are consistent with those reported by Diehl et al., who found that an ALC $>2 \times 10^{9} / \mathrm{L}$ at baseline was associated with grade 2 or higher irAEs (31). IrAEs are autoimmune and autoinflammatory disorders caused by a hyperreactive immune system after the upregulation of $\mathrm{T}$ cell immune function after treatment. It is possible that a higher level of pretreatment ALC improves the potential antitumor ability of $\mathrm{T}$ cells and their hyper-reactive ability to immunotherapy (32). Similarly, lower ALC has been reported to be associated with reduced multiple sclerosis disease activity, which is consistent with our cognition of irAEs and autoimmune diseases (33). As a biomarker, ALC value is directly presented in the lab reports and needless to calculate compared to PLR or NLR, which is easier to be used by clinicians.

Our results showed negative relationship between high NLR, high PLR and survival, which is consistent with former studies $(34,35)$. We also showed that a high 
pretreatment PNI is related to improved OS. Additionally, a high pretreatment PNI was also related to improved PFS in patients receiving chemoimmunotherapy. The PNI is a simple way to assess perioperative nutritional conditions and immunological status (36). In a study of Peng et al., baseline PNI $\geq 45$ was independently associated with both better PFS and longer OS in advanced lung cancer patients treated with PD-1 inhibitors (35). Johannet et al. found that a low baseline PNI was associated with a decreased response rate, shorter PFS and OS among patients with various types of cancer, including melanoma, lung cancer, and others malignancies treated with anti-PD-(L) 1 or anti-CTLA-4 antibodies (37). Our results provide further evidence that PNI represents a nutritional condition predicting response to chemoimmunotherapy. PNI also serves as a measure of a patient's immunological status (38), which plays an important role in a patient's response to immunotherapy.

IL-6 is closely involved in tumor immune-evasion and negatively correlated with prognosis in cancer patients (39). In line with this finding, our results showed that a high pretreatment IL-6 level was associated with shortened OS. There is a body of evidence that suggests that high pretreatment IL-6 is associated with a poor survival in patients undergoing treatment with ipilimumab (40-42). In the Checkmate-064, -066, and -067 trials, higher pretreatment IL-6 levels were associated with shortened OS in melanoma patients (43). Notably, the cutoff value of the Checkmate-064 study ( 15.1 and $12.7 \mathrm{pg} / \mathrm{mL}$ ) was quite close to the cutoff value used in our study $(13.8,11.3$ and $11.85 \mathrm{pg} / \mathrm{mL}$ ). This further supports the credibility of our findings and suggests that an IL-6 level around this interval is of prognostic significance. Low pretreatment IL-6 serum levels were also shown to be an independent risk factor for irAEs in malignant melanoma patients treated with the anti-CTLA-4 antibody (44). The potential role of serum IL-6 in patients with advanced lung cancer receiving ICI might be related to a stimulation of tumor cell proliferation, survival, and invasiveness through the IL-6/JAK/STAT3 pathway. Moreover, IL-6 and JAK/STAT3 signaling is often hyperactivated in tumor-infiltrating immune cells thus leading to downmodulation of antitumor immunity and contributing to a highly immunosuppressive tumor microenvironment (45).

An increased NLR $(\geq 2)$ during immunotherapy was reported to be a negative prognostic factor in patients with advanced cancers (46). Our results showed an association of increased on-treatment IL-6 and poor OS in the whole population. Moreover, the KM analysis showed best OS in patients with stable IL-6 group. This may be due to the small sample of patients. A decrease in IL-6 levels was earlier found to be associated with improved PFS in advanced lung cancer patients treated with PD-1 antibodies (28). In the Checkmate-064 study, increased serum IL-6 correlated with reduced survival (43). We failed to identify any relationship between changes in peripheral blood cell parameters and survival. This may be because our cohort comprised patients treated with both immunotherapy and chemoimmunotherapy. Chemotherapy is known to affect peripheral blood cell counts due to hematotoxicity. In our cohort, no information was available regarding the administration of granulocyte colony stimulating factor (GCSF) or thrombopoietin and blood products. Thus, we cannot exclude this potential confounder. Therefore, prospective studies are clearly needed to clarify this issue.

This study has several limitations. First, its retrospective nature and limited number of patients may have introduced case selection bias and restricted the generalizability of the results. Second, the patients received immunotherapy-based treatment at different lines of therapy. Thus, the degree of baseline inflammation may have been affected by previous treatments. Despite these limitations, an interesting finding of this study was the similarity of risk factors for survival in patients treated with both immunotherapy and immunochemotherapy. These results extend our understanding of the relationship between peripheral blood parameters and clinical outcomes after immunotherapybased treatment. Further studies are needed to validate our results and better establish cut-points for rigorous clinical implementation.

\section{Conclusions}

A high pretreatment ALC can be a predictor of increased risk of irAEs in patients with advanced NSCLC receiving immunotherapy-based treatments. In addition, high PNI and low IL-6 are correlated with improved survival. Blood-based markers may serve as reliable predictors of immune-related toxicity and survival outcomes in patients with advanced NSCLC treated with immunotherapy or chemoimmunotherapy.

\section{Acknowledgments}

The authors appreciate the academic support from the AME Lung Cancer Collaborative Group. Funding: None. 


\section{Footnote}

Reporting Checklist: The authors have completed the REMARK reporting checklist. Available at https://dx.doi. org/10.21037/tlcr-21-710

Data Sharing Statement: Available at https://dx.doi. org/10.21037/tlcr-21-710

Conflicts of Interest: All authors have completed the ICMJE uniform disclosure form (available at https:// dx.doi.org/10.21037/tlcr-21-710). Dr. JJ reported that he attained consulting fees from AstraZeneca, BMS, MSD, Pfizer, Roche, and got travel support form Roche, Pfizer, Boehringer. The other authors have no conflicts of interest to declare.

Ethical Statement: The authors are accountable for all aspects of the work in ensuring that questions related to the accuracy or integrity of any part of the work are appropriately investigated and resolved. This study was performed in accordance with the principles for Good Clinical Practice and the Declaration of Helsinki (as revised in 2013). The study was approved by the Internal Review Board of PUMCH on August 31, 2020 (protocol number SK-135) and individual consent for this retrospective analysis was waived.

Open Access Statement: This is an Open Access article distributed in accordance with the Creative Commons Attribution-NonCommercial-NoDerivs 4.0 International License (CC BY-NC-ND 4.0), which permits the noncommercial replication and distribution of the article with the strict proviso that no changes or edits are made and the original work is properly cited (including links to both the formal publication through the relevant DOI and the license). See: https://creativecommons.org/licenses/by-nc-nd/4.0/.

\section{References}

1. Gadgeel S, Rodríguez-Abreu D, Speranza G, et al. Updated Analysis From KEYNOTE-189: Pembrolizumab or Placebo Plus Pemetrexed and Platinum for Previously Untreated Metastatic Nonsquamous Non-Small-Cell Lung Cancer. J Clin Oncol 2020;38:1505-17.

2. Pacheco JM. KEYNOTE-042: is lowering the PD-L1 threshold for first-line pembroli-zumab monotherapy a good idea? Transl Lung Cancer Res 2019;8:723-7.
3. Paz-Ares L, Ciuleanu TE, Cobo M, et al. First-line nivolumab plus ipilimumab combined with two cycles of chemotherapy in patients with non-small-cell lung cancer (CheckMate 9LA): an international, randomised, openlabel, phase 3 trial. Lancet Oncol 2021;22:198-211.

4. Herbst RS, Garon EB, Kim DW, et al. Long-Term Outcomes and Retreatment Among Patients With Previously Treated, Programmed Death-Ligand 1Positive, Advanced Non-Small-Cell Lung Cancer in the KEYNOTE-010 Study. J Clin Oncol 2020;38:1580-90.

5. Antonia SJ, Borghaei H, Ramalingam SS, et al. Four-year survival with nivolumab in patients with previously treated advanced non-small-cell lung cancer: a pooled analysis. Lancet Oncol 2019;20:1395-408.

6. Antonia SJ, Villegas A, Daniel D, et al. Durvalumab after Chemoradiotherapy in Stage III Non-Small-Cell Lung Cancer. N Engl J Med 2017;377:1919-29.

7. Reck M, Rodríguez-Abreu D, Robinson AG, et al. Updated Analysis of KEYNOTE-024: Pembrolizumab Versus Platinum-Based Chemotherapy for Advanced NonSmall-Cell Lung Cancer With PD-L1 Tumor Proportion Score of 50\% or Greater. J Clin Oncol 2019;37:537-46.

8. Socinski MA, Jotte RM, Cappuzzo F, et al. Atezolizumab for First-Line Treatment of Metastatic Nonsquamous NSCLC. N Engl J Med 2018;378:2288-301.

9. Tarhini AA, Zahoor $\mathrm{H}$, Lin $\mathrm{Y}$, et al. Baseline circulating IL-17 predicts toxicity while TGF- $\beta 1$ and IL-10 are prognostic of relapse in ipilimumab neoadjuvant therapy of melanoma. J Immunother Cancer 2015;3:39.

10. Nakamura Y, Tanaka R, Maruyama H, et al. Correlation between blood cell count and outcome of melanoma patients treated with anti-PD-1 antibodies. Jpn J Clin Oncol 2019;49:431-7.

11. Shahabi V, Berman D, Chasalow SD, et al. Gene expression profiling of whole blood in ipilimumab-treated patients for identification of potential biomarkers of immune-related gastrointestinal adverse events. J Transl Med 2013;11:75.

12. Lim SY, Lee JH, Gide TN, et al. Circulating Cytokines Predict Immune-Related Toxicity in Melanoma Patients Receiving Anti-PD-1-Based Immunotherapy. Clin Cancer Res 2019;25:1557-63.

13. Negrao MV, Lam VK, Reuben A, et al. PD-L1 Expression, Tumor Mutational Burden, and Cancer Gene Mutations Are Stronger Predictors of Benefit from Immune Checkpoint Blockade than HLA Class I Genotype in Non-Small Cell Lung Cancer. J Thorac Oncol 2019;14:1021-31.

14. Le DT, Durham JN, Smith KN, et al. Mismatch repair 
deficiency predicts response of solid tumors to PD-1 blockade. Science 2017;357:409-13.

15. Chu X, Zhao J, Zhou J, et al. Association of baseline peripheral-blood eosinophil count with immune checkpoint inhibitor-related pneumonitis and clinical outcomes in patients with non-small cell lung cancer receiving immune checkpoint inhibitors. Lung Cancer 2020;150:76-82.

16. Ferrucci PF, Ascierto PA, Pigozzo J, et al. Baseline neutrophils and derived neutrophil-to-lymphocyte ratio: prognostic relevance in metastatic melanoma patients receiving ipilimumab. Ann Oncol 2018;29:524.

17. Khoja L, Atenafu EG, Templeton A, et al. The full blood count as a biomarker of outcome and toxicity in ipilimumab-treated cutaneous metastatic melanoma. Cancer Med 2016;5:2792-9.

18. Watanabe I, Kanauchi N, Watanabe H. Preoperative prognostic nutritional index as a predictor of outcomes in elderly patients after surgery for lung cancer. Jpn J Clin Oncol 2018;48:382-7.

19. Harlin H, Meng Y, Peterson AC, et al. Chemokine expression in melanoma metastases associated with CD8+ T-cell recruitment. Cancer Res 2009;69:3077-85.

20. Hoejberg L, Bastholt L, Johansen JS, et al. Serum interleukin-6 as a prognostic biomarker in patients with metastatic melanoma. Melanoma Res 2012;22:287-93.

21. Lutgendorf SK, Weinrib AZ, Penedo F, et al. Interleukin-6, cortisol, and depressive symptoms in ovarian cancer patients. J Clin Oncol 2008;26:4820-7.

22. Knüpfer H, Preiss R. Serum interleukin-6 levels in colorectal cancer patients--a summary of published results. Int J Colorectal Dis 2010;25:135-40.

23. Zhang $\mathrm{C}$, Xin H, Zhang W, et al. CD5 Binds to Interleukin-6 and Induces a Feed-Forward Loop with the Transcription Factor STAT3 in B Cells to Promote Cancer. Immunity 2016;44:913-23.

24. Linnskog R, Jönsson G, Axelsson L, et al. Interleukin-6 drives melanoma cell motility through p38 $\alpha$-MAPKdependent up-regulation of WNT5A expression. Mol Oncol 2014;8:1365-78.

25. Tsukamoto H, Fujieda K, Hirayama M, et al. Soluble IL6R Expressed by Myeloid Cells Reduces Tumor-Specific Th1 Differentiation and Drives Tumor Progression. Cancer Res 2017;77:2279-91.

26. Ara T, Nakata R, Sheard MA, et al. Critical role of STAT3 in IL-6-mediated drug resistance in human neuroblastoma. Cancer Res 2013;73:3852-64.

27. Drobni ZD, Zafar A, Zubiri L, et al. Decreased Absolute
Lymphocyte Count and Increased Neutrophil/Lymphocyte Ratio With Immune Checkpoint Inhibitor-Associated Myocarditis. J Am Heart Assoc 2020;9:e018306.

28. Keegan A, Ricciuti B, Garden P, et al. Plasma IL-6 changes correlate to PD-1 inhibitor responses in NSCLC. J Immunother Cancer 2020;8:e00678.

29. Bagley SJ, Kothari S, Aggarwal C, et al. Pretreatment neutrophil-to-lymphocyte ratio as a marker of outcomes in nivolumab-treated patients with advanced non-small-cell lung cancer. Lung Cancer 2017;106:1-7.

30. Eisenhauer EA, Therasse P, Bogaerts J, et al. New response evaluation criteria in solid tumours: revised RECIST guideline (version 1.1). Eur J Cancer 2009;45:228-47.

31. Diehl A, Yarchoan M, Hopkins A, et al. Relationships between lymphocyte counts and treatment-related toxicities and clinical responses in patients with solid tumors treated with PD-1 checkpoint inhibitors. Oncotarget 2017;8:114268-80.

32. Schietinger A, Greenberg PD. Tolerance and exhaustion: defining mechanisms of $T$ cell dysfunction. Trends Immunol 2014;35:51-60.

33. Garbo R, Lorenzut S, Del Negro I, et al. Lower lymphocyte counts and older age are associated with reduced multiple sclerosis disease activity during dimethyl fumarate treatment. Mult Scler Relat Disord 2021;49:102781.

34. Park CK, Oh HJ, Kim MS, et al. Comprehensive analysis of blood-based biomarkers for predicting immunotherapy benefits in patients with advanced non-small cell lung cancer. Transl Lung Cancer Res 2021;10:2103-17.

35. Peng L, Wang Y, Liu F, et al. Peripheral blood markers predictive of outcome and immune-related adverse events in advanced non-small cell lung cancer treated with PD-1 inhibitors. Cancer Immunol Immunother 2020;69:1813-22.

36. Zhang J, Zhang Y, Yv X, et al. Prognostic value of combined preoperative prognostic nu-tritional index and neutrophil to lymphocyte ratio in esophageal squamous cell carcinoma. Transl Cancer Res 2020;9:5117-27.

37. Johannet P, Sawyers A, Qian Y, et al. Baseline prognostic nutritional index and changes in pretreatment body mass index associate with immunotherapy response in patients with advanced cancer. J Immunother Cancer 2020;8:e001674.

38. Mantovani A, Allavena P, Sica A, et al. Cancer-related inflammation. Nature 2008;454:436-44.

39. Chen M, Ye X, Wang R, et al. Research progress of cancer stem cells and IL-6/STAT3 signaling pathway 
in esophageal adenocarcinoma. Transl Cancer Res 2020;9:363-71.

40. Tjin EP, Krebbers G, Meijlink KJ, et al. Immune-escape markers in relation to clinical outcome of advanced melanoma patients following immunotherapy. Cancer Immunol Res 2014;2:538-46.

41. Hardy-Werbin M, Rocha P, Arpi O, et al. Serum cytokine levels as predictive biomarkers of benefit from ipilimumab in small cell lung cancer. Oncoimmunology 2019;8:e1593810.

42. Bjoern J, Juul Nitschke N, Zeeberg Iversen T, et al. Immunological correlates of treatment and response in stage IV malignant melanoma patients treated with Ipilimumab. Oncoimmunology 2015;5:e1100788.

43. Laino AS, Woods D, Vassallo M, et al. Serum interleukin-6 and C-reactive protein are associated with survival in melanoma patients receiving immune checkpoint inhibition. J Immunother Cancer 2020;8:e000842.

44. Valpione S, Pasquali S, Campana LG, et al. Sex and interleukin-6 are prognostic factors for autoimmune toxicity following treatment with anti-CTLA4 blockade. J Transl Med 2018;16:94.

45. Johnson DE, O'Keefe RA, Grandis JR. Targeting the IL-6/JAK/STAT3 signalling axis in cancer. Nat Rev Clin Oncol 2018;15:234-48.

46. Ota Y, Takahari D, Suzuki T, et al. Changes in the neutrophil-to-lymphocyte ratio during nivolumab monotherapy are associated with gastric cancer survival. Cancer Chemother Pharmacol 2020;85:265-72.

Cite this article as: Shi Y, Liu X, Liu J, Zhang D, Liu X, Yue Y, Zhou Q, Gao X, Chen M, Xu Y, Zhao J, Zhong W, Provencio M, Jassem J, Williams TM, Seeber A, Kocher F, Wang M. Correlations between peripheral blood biomarkers and clinical outcomes in advanced non-small cell lung cancer patients who received immunotherapy-based treatments. Transl Lung Cancer Res 2021;10(12):4477-4493. doi: 10.21037/tlcr-21-710 


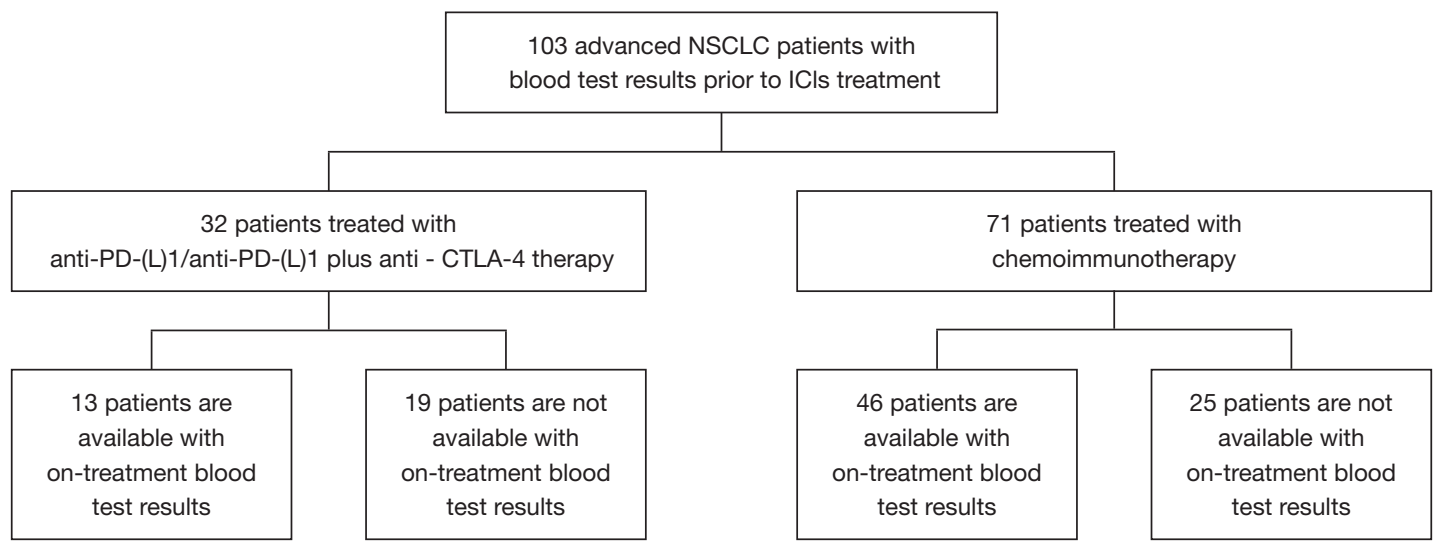

Figure S1 Patient enrollment in this study. NSCLC, non-small cell lung cancer; ICIs, immune checkpoint inhibitors; PD-(L)1, program death-(ligand) 1; CTLA-4, cytotoxic T lymphocyte associated protein-4.

Table S1 Baseline peripheral blood parameters' associated risk of best overall response in the whole population $(\mathrm{n}=103)$

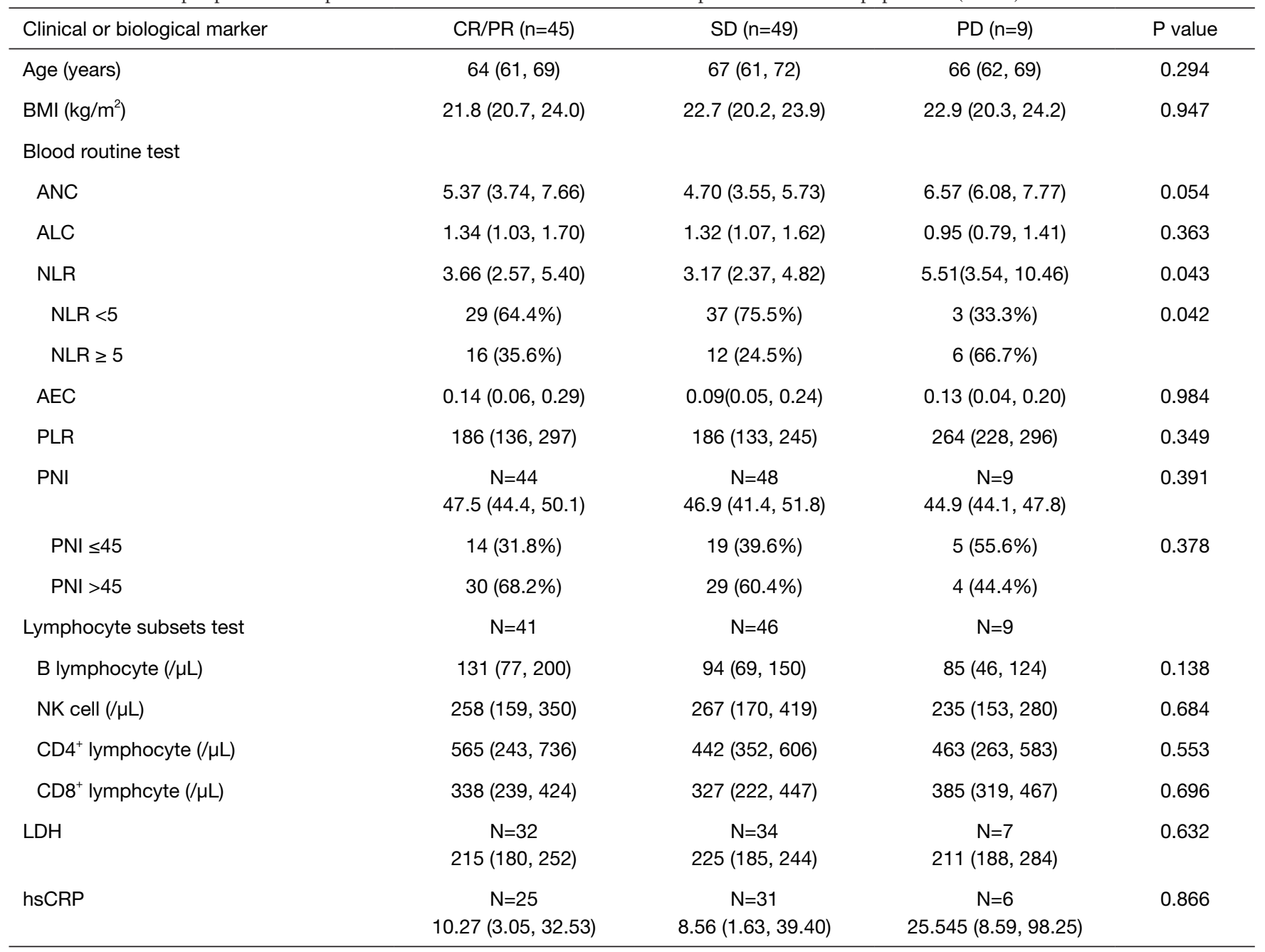

Table S1 (continued) 
Table S1 (continued)

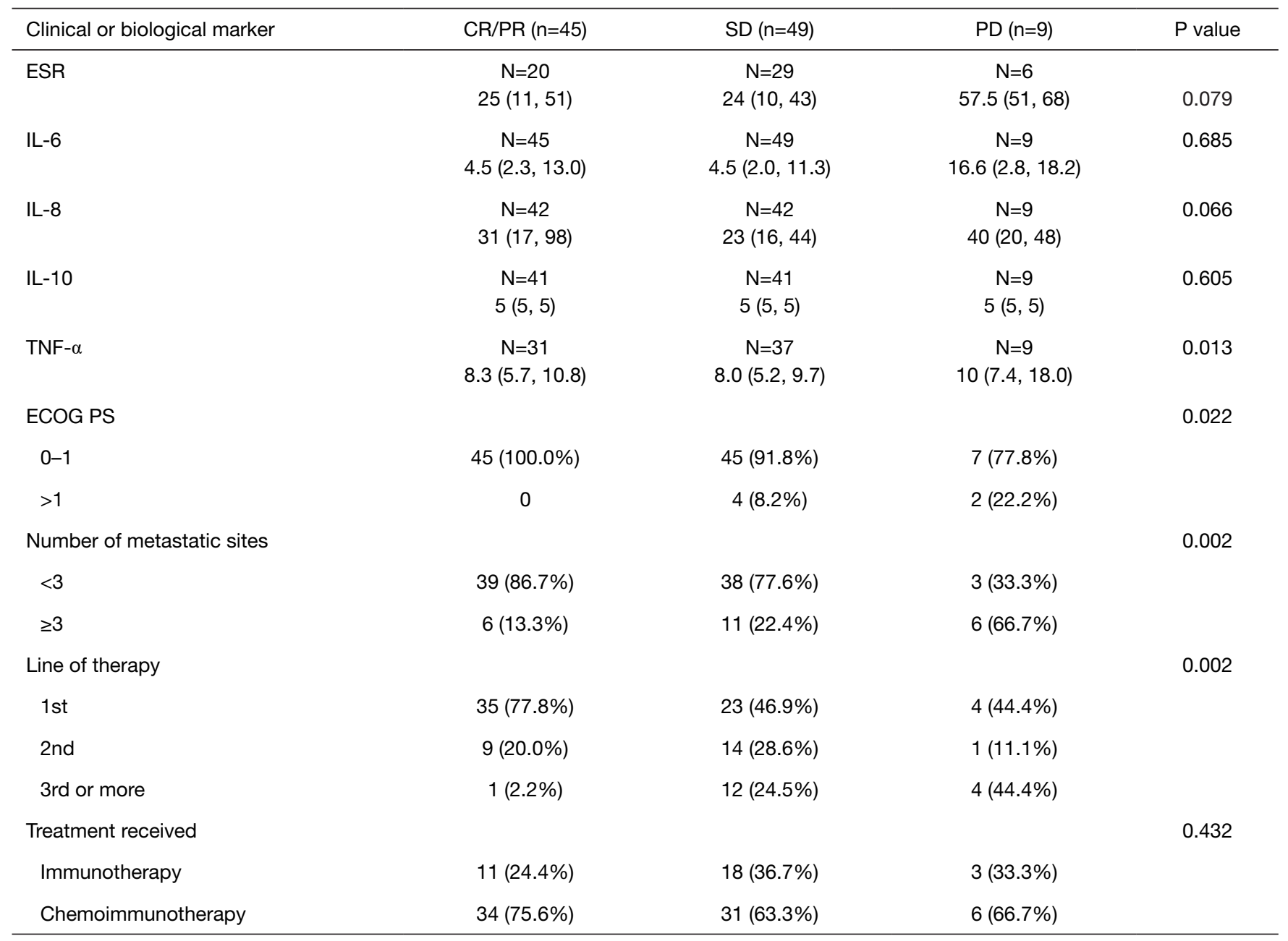

CR, complete response; PR, partial response; SD, stable disease; PD, progressive disease; BMI, body mass index; ANC, absolute neutrophil count; ALC, absolute lymphocyte count; NLR, neutrophil-lymphocyte ratio; AEC, absolute eosinophils count; PLR, platelet-lymphocyte ratio; PNI, prognostic nutrition index; NK, natural killer cell; LDH, lactate dehydrogenase; hsCRP, hyper-sensitive C-reactive protein; ESR, erythrocyte sedimentation rate; IL-6, interleukin-6; IL-8, interleukin-8; IL-10, interleukin-10; TNF- $\alpha$, tumor necrosis factor $\alpha$; ECOG PS, Eastern Cooperative Oncology Group performance status. 
Table S2 Multivariate analyses of best overall response in the whole population $(\mathrm{n}=103)$

\begin{tabular}{|c|c|c|c|c|c|c|}
\hline Variable & \multicolumn{3}{|c|}{ PR vs. PD } & \multicolumn{3}{|c|}{ SD vs. PD } \\
\hline NLR & 0.946 & $0.662-1.352$ & 0.759 & 0.783 & $0.528-1.162$ & 0.224 \\
\hline AEC & 1.064 & $0.168-6.727$ & 0.948 & 0.570 & $0.087-3.721$ & 0.557 \\
\hline PLR & 1.003 & $0.991-1.015$ & 0.673 & 0.997 & $0.986-1.009$ & 0.668 \\
\hline IL-6 & 0.972 & $0.855-1.104$ & 0.658 & 1.035 & $0.925-1.159$ & 0.548 \\
\hline IL-8 & 1.000 & $0.982-1.018$ & 0.992 & 0.993 & $0.977-1.009$ & 0.385 \\
\hline TNF- $\alpha$ & 0.983 & $0.870-1.112$ & 0.789 & 1.007 & $0.915-1.108$ & 0.892 \\
\hline Treatment line $(>1)$ & 3.034 & $0.372-24.713$ & 0.300 & 1.102 & $0.149-8.178$ & 0.924 \\
\hline
\end{tabular}

', there is no ECOG >1 patients in PR group. NLR, neutrophil-lymphocyte ratio; AEC, absolute eosinophils count; PLR, platelet-lymphocyte ratio; PNI, prognostic nutrition index; IL-6, interleukin-6; IL-8, interleukin-8; TNF- $\alpha$, tumor necrosis factor $\alpha$; ECOG PS, Eastern Cooperative Oncology Group performance status. 
Table S3 Baseline peripheral blood parameters' associated risk of best overall response in patients receiving anti-PD-1/PD-L1 monotherapy or anti-PD-1/PD-L1 plus anti CTLA-4 (n=32)

\begin{tabular}{|c|c|c|c|c|}
\hline Clinical or biological marker & CR/PR $(n=11)$ & $S D(n=18)$ & $\mathrm{PD}(\mathrm{n}=3)$ & $P$ value \\
\hline ANC & $3.800(3.12,4.93)$ & $4.115(2.97,5.08)$ & $6.260(6.08,7.77)$ & 0.350 \\
\hline ALC & $1.370(1.10,1.88)$ & $1.350(1.07,1.62)$ & $1.410(0.81,1.80)$ & 0.880 \\
\hline NLR & $2.690(2.33,3.22)$ & $2.740(2.01,5.88)$ & $5.510(3.38,7.73)$ & 0.616 \\
\hline PLR & $142.860(119.70,226.13)$ & $189.385(124.65,275.70)$ & $228.370(188.89,371.60)$ & 0.547 \\
\hline PNI & $\begin{array}{c}\mathrm{N}=11 \\
48.950(47.50,51.50)\end{array}$ & $\begin{array}{c}\mathrm{N}=18 \\
46.625(40.50,52.15)\end{array}$ & $\begin{array}{c}\mathrm{N}=3 \\
47.050(44.05,48.00)\end{array}$ & 0.296 \\
\hline \multicolumn{5}{|l|}{ Lymphocyte subsets } \\
\hline CD8 ${ }^{+}$lymphocyte & $363.0(239,404)$ & $316.0(222,441)$ & $439.0(385,485)$ & 0.626 \\
\hline LDH & $\begin{array}{c}\mathrm{N}=7 \\
188.0(170,202)\end{array}$ & $\begin{array}{c}\mathrm{N}=12 \\
216.0(177,240)\end{array}$ & $\begin{array}{c}N=2 \\
273.5(263,284)\end{array}$ & 0.147 \\
\hline hsCRP & $\begin{array}{c}\mathrm{N}=6 \\
17.915(3.05,53.92)\end{array}$ & $\begin{array}{c}\mathrm{N}=12 \\
5.500(1.84,22.56)\end{array}$ & $\begin{array}{c}\mathrm{N}=2 \\
69.865(41.48,98.25)\end{array}$ & 0.311 \\
\hline ESR & $\begin{array}{c}N=6 \\
25.0(6,32)\end{array}$ & $\begin{array}{c}N=11 \\
24.0(10,52)\end{array}$ & $\begin{array}{c}N=2 \\
84.0(51,117)\end{array}$ & 0.029 \\
\hline IL-6 & $\begin{array}{c}\mathrm{N}=11 \\
4.70(2.0,22.8)\end{array}$ & $\begin{array}{c}\mathrm{N}=18 \\
4.95(3.2,11.9)\end{array}$ & $\begin{array}{c}\mathrm{N}=3 \\
18.20(16.6,33.9)\end{array}$ & 0.595 \\
\hline
\end{tabular}

CR, complete response; PR, partial response; SD, stable disease; PD, progressive disease; ANC, absolute neutrophil count; ALC, absolute lymphocyte count; NLR, neutrophil-lymphocyte ratio; AEC, absolute eosinophils count; PLR, platelet-lymphocyte ratio; PNI, prognostic nutrition index; NK, natural killer cell; LDH, lactate dehydrogenase; hsCRP, hyper-sensitive C-reactive protein; ESR, erythrocyte sedimentation rate; IL-6, interleukin-6; IL-8, interleukin-8; IL-10, interleukin-10; NA, not available; TNF- $\alpha$, tumor necrosis factor $\alpha$. 
Table S4 Baseline peripheral blood parameters' associated risk of best overall response in patients receiving chemoimmunotherapy (n=71)

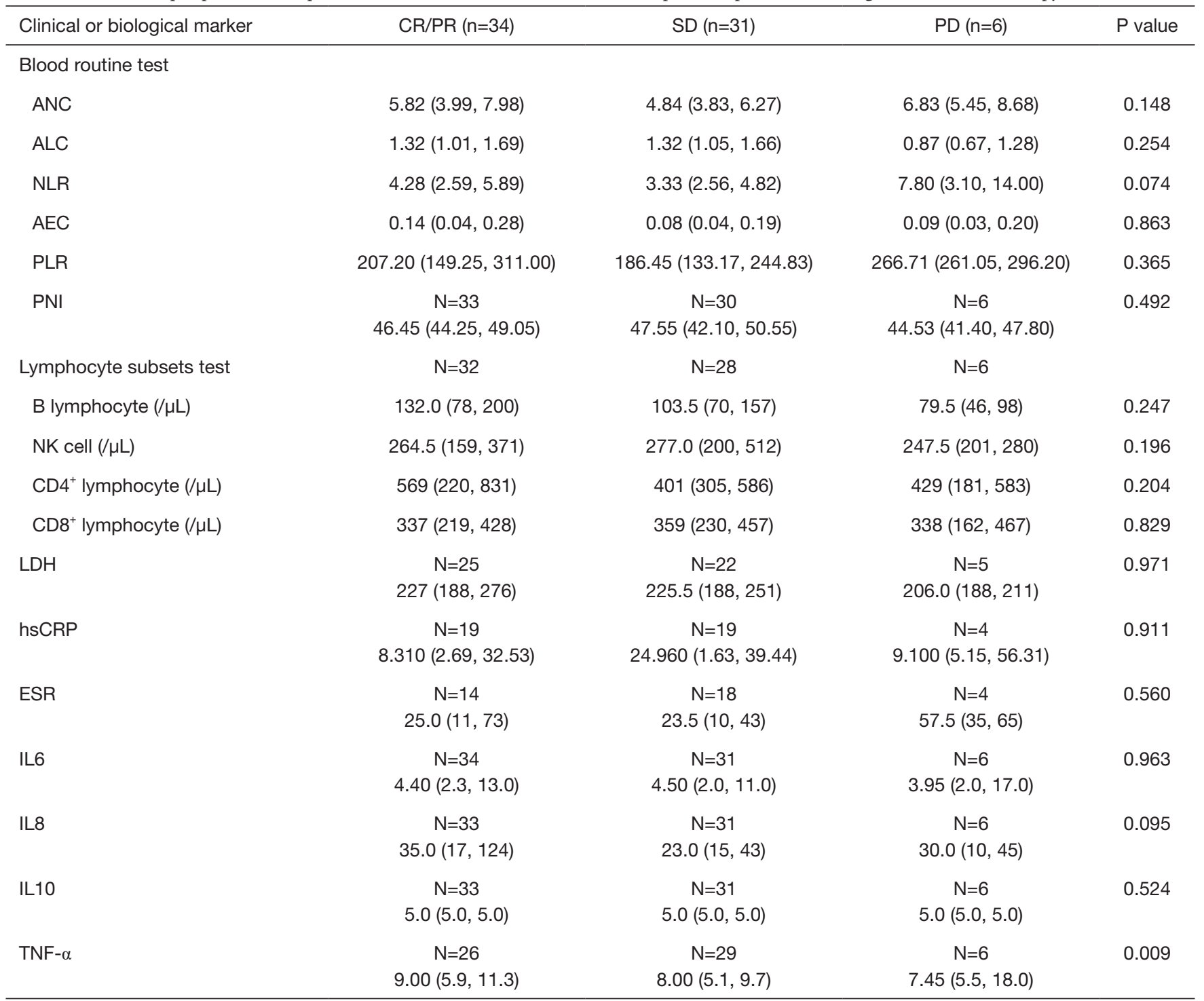

CR, complete response; PR, partial response; SD, stable disease; PD, progressive disease; ANC, absolute neutrophil count; ALC, absolute lymphocyte count; NLR, neutrophil-lymphocyte ratio; AEC, absolute eosinophils count; PLR, platelet-lymphocyte ratio; PNI, prognostic nutrition index; NK, natural killer cell; LDH, lactate dehydrogenase; hsCRP, hyper-sensitive C-reactive protein; ESR, erythrocyte sedimentation rate; IL-6, interleukin-6; IL-8, interleukin-8; IL-10, interleukin-10; TNF- $\alpha$, tumor necrosis factor $\alpha$. 
Table S5 Baseline peripheral blood parameters' associated risk of best overall response in patients receiving first-line chemoimmunotherapy ( $\mathrm{n}=53$ )

\begin{tabular}{|c|c|c|c|c|}
\hline Clinical or biological marker & CR/PR (n=31) & $S D(n=19)$ & $P D(n=3)$ & $P$ value \\
\hline \multicolumn{5}{|l|}{ Blood routine test } \\
\hline ANC & $5.89(4.455,8.125)$ & $5.23(3.905,6.445)$ & $6.57(5.45,6.58)$ & 0.276 \\
\hline ALC & $1.29(1.02,1.69)$ & $1.19(1.035,1.57)$ & $1.28(0.67,1.76)$ & 0.933 \\
\hline NLR & $4.95(2.82,6.15)$ & $3.33(2.80,6.28)$ & $5.13(3.10,10.57)$ & 0.553 \\
\hline PLR & $214.4(152.65,304.88)$ & $196.52(165.98,281.82)$ & $264.06(183.52,505.97)$ & 0.664 \\
\hline PNI & $\begin{array}{c}\mathrm{N}=30 \\
45.88(43.65,48.99)\end{array}$ & $\begin{array}{c}\mathrm{N}=18 \\
46.075(39.375,50.25)\end{array}$ & $\begin{array}{c}\mathrm{N}=3 \\
47.80(41.40,48.35)\end{array}$ & 0.978 \\
\hline Lymphocyte subsets test & $\mathrm{N}=27$ & $N=16$ & $\mathrm{~N}=3$ & \\
\hline CD8 ${ }^{+}$T lymphocyte $(/ \mu \mathrm{L})$ & $336(243.5,426)$ & $284.5(200.75,417)$ & $467(319,471)$ & 0.303 \\
\hline LDH & $\begin{array}{c}\mathrm{N}=23 \\
220(185,251.5)\end{array}$ & $\begin{array}{c}\mathrm{N}=14 \\
226(215.25,257.75)\end{array}$ & $\begin{array}{l}\mathrm{N}=2 \\
\mathrm{NA}\end{array}$ & 0.310 \\
\hline hsCRP & $\begin{array}{c}\mathrm{N}=17 \\
8.31(3.11,31.14)\end{array}$ & $\begin{array}{c}\mathrm{N}=12 \\
31.42(4.38,60.135)\end{array}$ & $\begin{array}{l}N=1 \\
9.61\end{array}$ & 0.495 \\
\hline ESR & $\begin{array}{c}\mathrm{N}=12 \\
25.0(11.08,74.5)\end{array}$ & $\begin{array}{c}\mathrm{N}=11 \\
28(19.5,48.5)\end{array}$ & $\begin{array}{l}\mathrm{N}=2 \\
\mathrm{NA}\end{array}$ & 0.971 \\
\hline IL6 & $\begin{array}{c}\mathrm{N}=31 \\
4.2(2.25,9.85)\end{array}$ & $\begin{array}{c}\mathrm{N}=19 \\
4.5(2,17.3)\end{array}$ & $\begin{array}{c}\mathrm{N}=3 \\
5.1(2.8,19.5)\end{array}$ & 0.824 \\
\hline
\end{tabular}

CR, complete response; PR, partial response; SD, stable disease; PD, progressive disease; ANC, absolute neutrophil count; ALC, absolute lymphocyte count; NLR, neutrophil-lymphocyte ratio; AEC, absolute eosinophils count; PLR, platelet-lymphocyte ratio; PNI, prognostic nutrition index; NK, natural killer cell; LDH, lactate dehydrogenase; NA, not available; hsCRP, hyper-sensitive C-reactive protein; ESR, erythrocyte sedimentation rate; IL-6, interleukin-6; IL-8, interleukin-8; IL-10, interleukin-10; TNF- $\alpha$, tumor necrosis factor $\alpha$. 
Table S6 Baseline peripheral blood parameters' associated risk of incidence of irAEs in patients receiving chemoimmunotherapy (n=71)

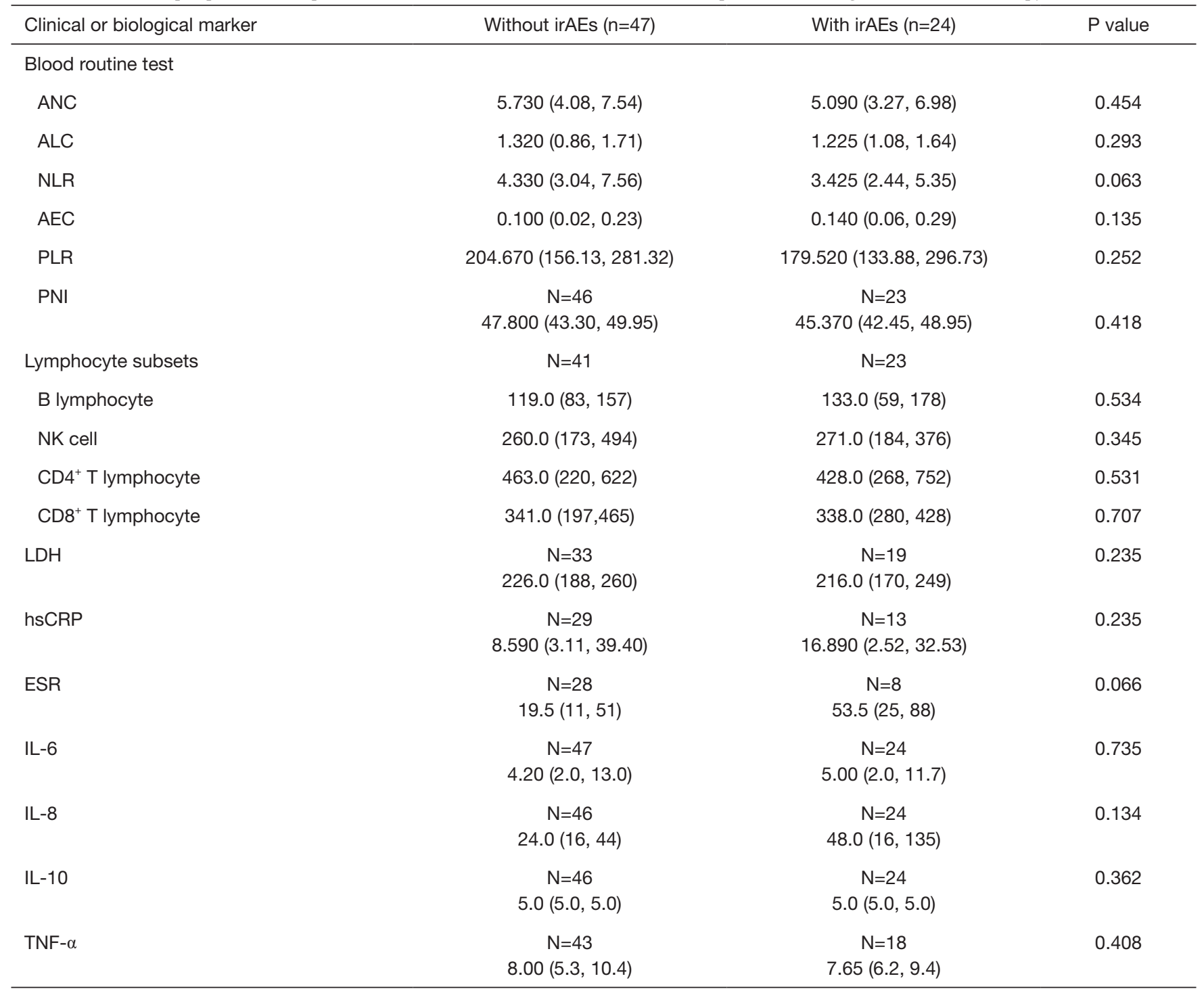

ANC, absolute neutrophil count; ALC, absolute lymphocyte count; NLR, neutrophil-lymphocyte ratio; AEC, absolute eosinophils count; PLR, platelet-lymphocyte ratio; PNI, prognostic nutrition index; NK cell, natural killer cell; LDH, lactate dehydrogenase; hsCRP, hyper-sensitive C-reactive protein; ESR, erythrocyte sedimentation rate; IL-6, interleukin-6; IL-8, interleukin-8; IL-10, interleukin-10; TNF- $\alpha$, tumor necrosis factor $\alpha$. 
Table S7 Baseline peripheral blood parameters' associated risk of incidence of irAEs in patients receiving chemoimmunotherapy as first-line therapy ( $\mathrm{n}=53)$

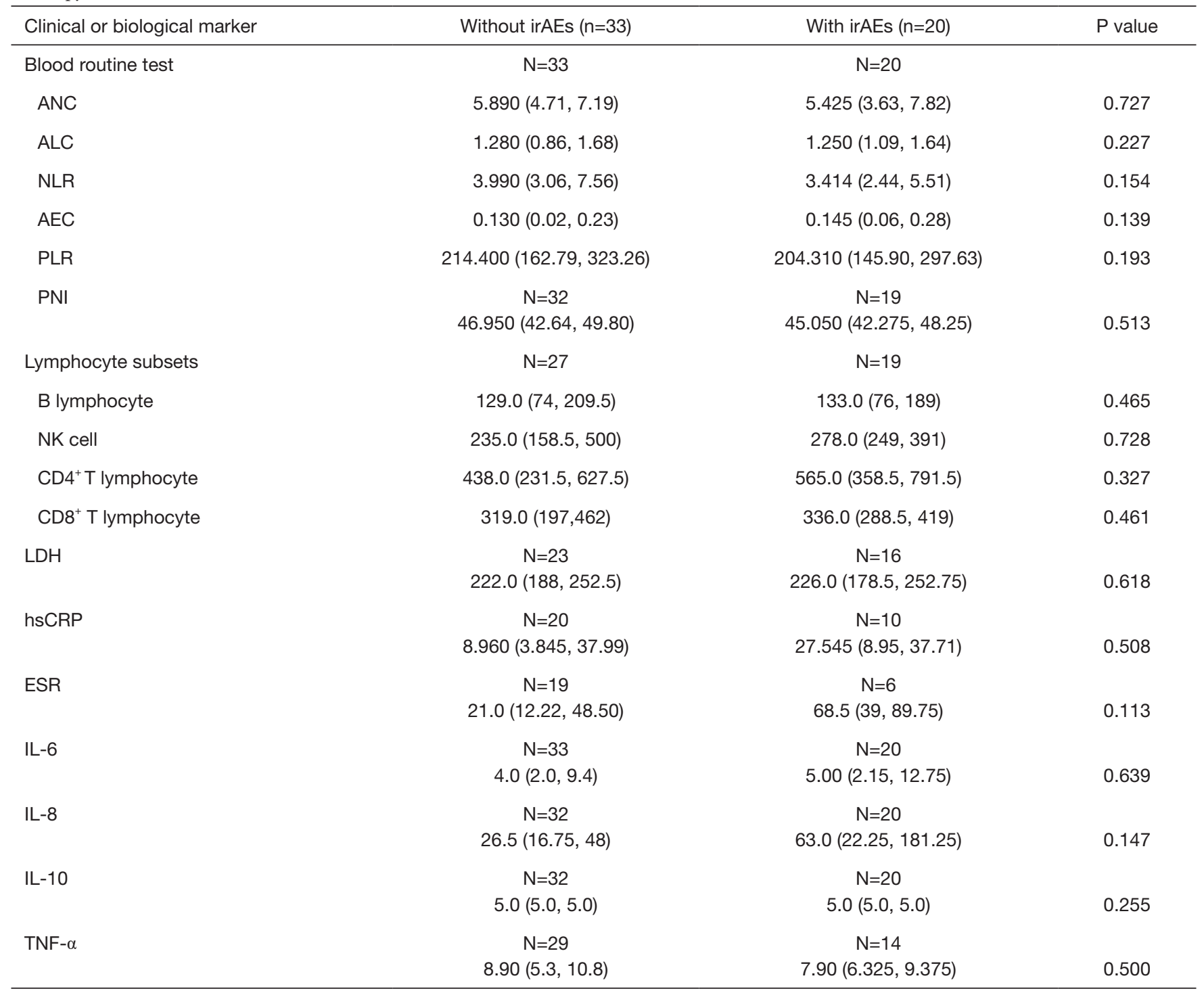

ANC, absolute neutrophil count; ALC, absolute lymphocyte count; NLR, neutrophil-lymphocyte ratio; AEC, absolute eosinophils count; PLR, platelet-lymphocyte ratio; PNI, prognostic nutrition index; NK cell, natural killer cell; LDH, lactate dehydrogenase; hsCRP, hyper-sensitive C-reactive protein; ESR, erythrocyte sedimentation rate; IL-6, interleukin-6; IL-8, interleukin-8; IL-10, interleukin-10; TNF- $\alpha$, tumor necrosis factor $\alpha$. 

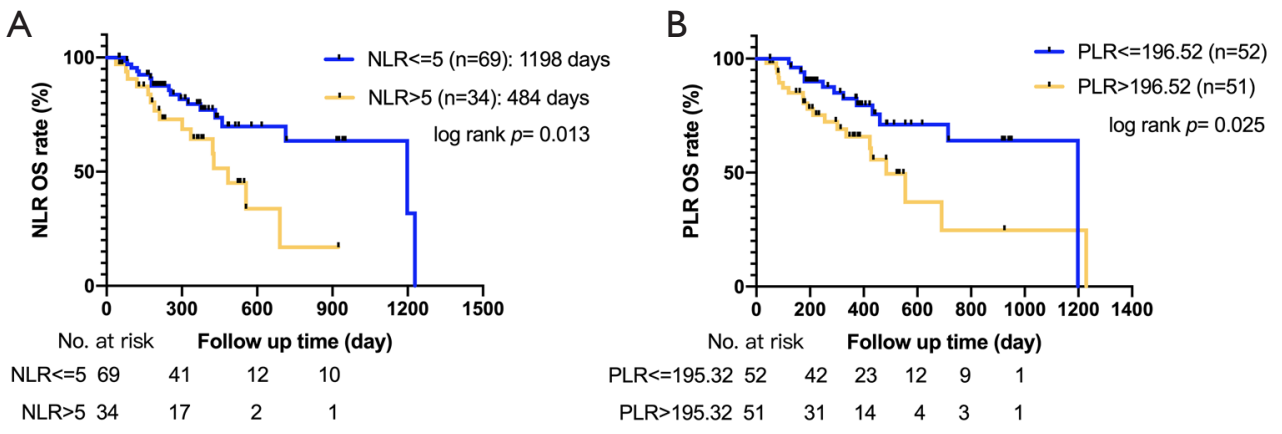

Figure S2 Kaplan-Meier analysis of OS for all patients ( $\mathrm{n}=103)$ with ICI-treated advanced NSCLC according to the NLR ( $\leq 5$ vs. $>5)$ (A) and the PLR ( $\leq$ median of 196.52 vs. >196.52) (B). The P values were calculated with the log rank test. OS, overall survival; ICI, immune checkpoint inhibitor; NSCLC, non-small cell lung cancer; NLR, neutrophil-to-lymphocyte ratio; PLR, platelet-to-lymphocyte ratio.

A

A

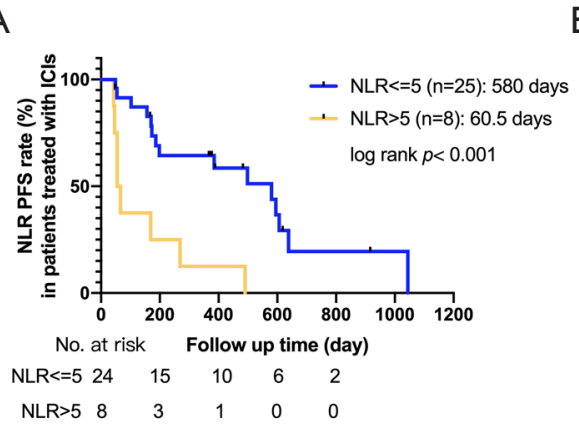

B $\frac{\mathscr{Q}}{\mathrm{O}} 100$

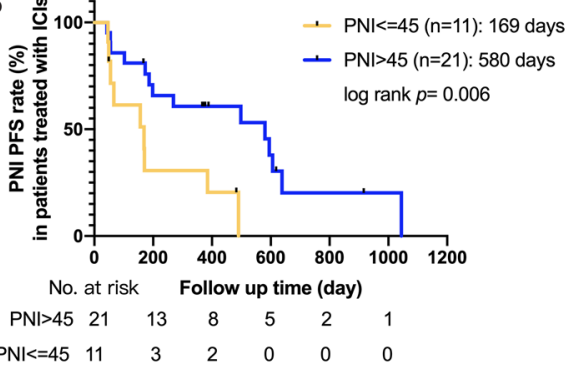

C
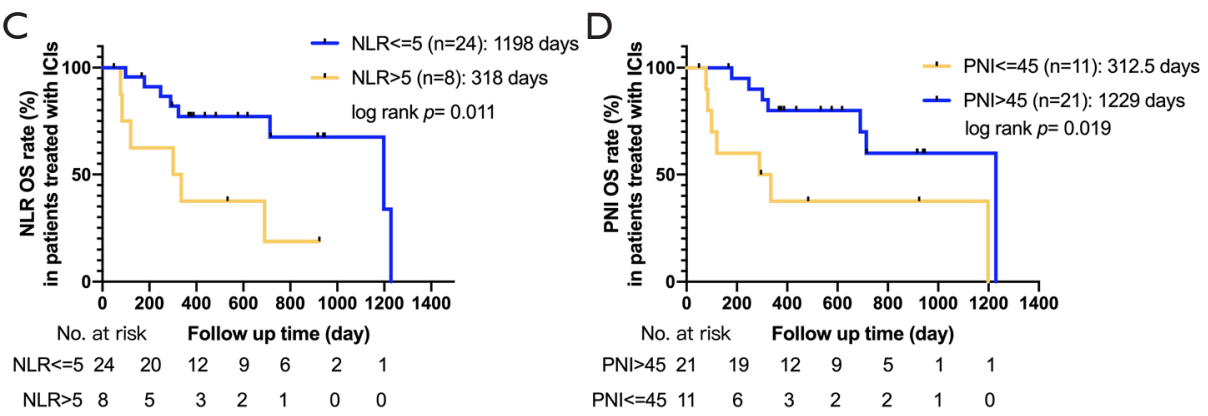

E

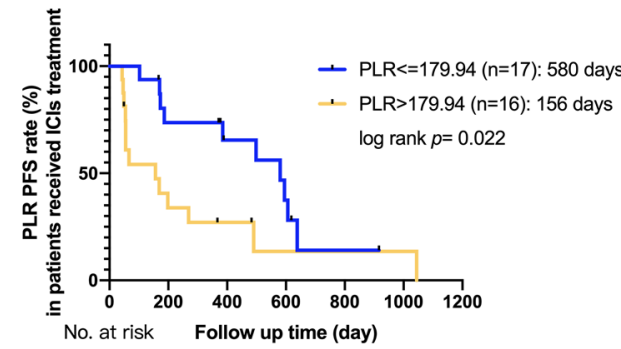

$\begin{array}{lllllll}\mathrm{PLR}<=179.94 & 16 & 11 & 7 & 4 & 1 & 0\end{array}$

$\begin{array}{lllllll}P L R>179.94 & 16 & 5 & 3 & 1 & 1 & 1\end{array}$

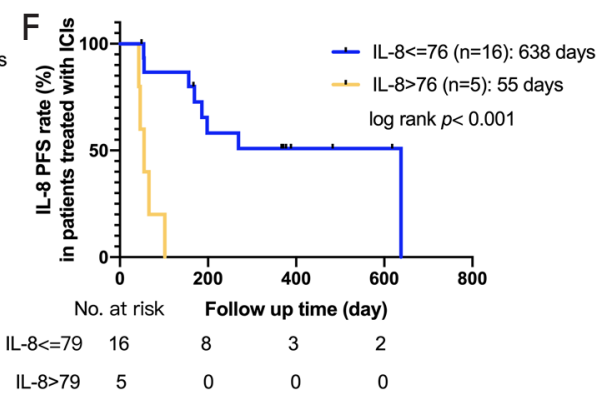

Figure S3 Kaplan-Meier analysis of PFS or OS for patients receiving immunotherapy only (n=32) according to the NLR $(\leq 5 v s .>5)(\mathrm{A}, \mathrm{C})$, the PNI ( $\leq 45 v s .>45)(\mathrm{B}, \mathrm{D})$, the PLR ( $\leq$ median of $179.94 v s .>179.94)(\mathrm{E})$, and the IL-8 ( $\leq 76 v s .>76)(\mathrm{F})$. The P values were calculated with the log rank test. PFS, progression-free survival; OS, overall survival; NLR, neutrophil-to-lymphocyte ratio; PNI, prognostic nutritional index; PLR, platelet-to-lymphocyte ratio; IL-8, interleukin-8. 

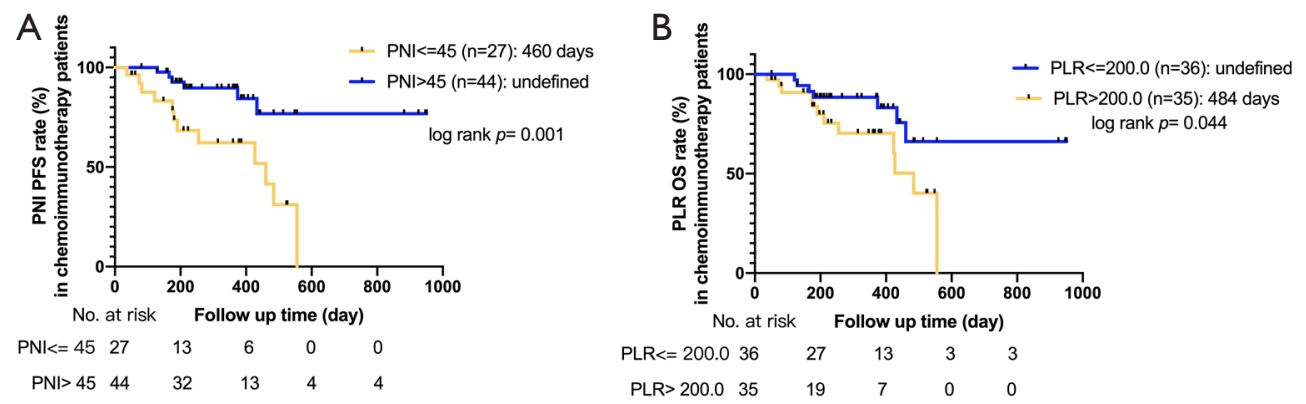

Figure S4 For patients receiving chemoimmunotherapy (n=71), Kaplan-Meier analysis of PFS according to the PNI ( $\leq 45 v s$. $>45)$ (A) and Kaplan-Meier analysis of OS according to the PLR ( $\leq 200 v s .>200)$ (B). The P values were calculated with the log rank test. PFS, progression free survival; PNI, prognostic nutritional index; OS, overall survival; PLR, platelet-to-lymphocyte ratio.
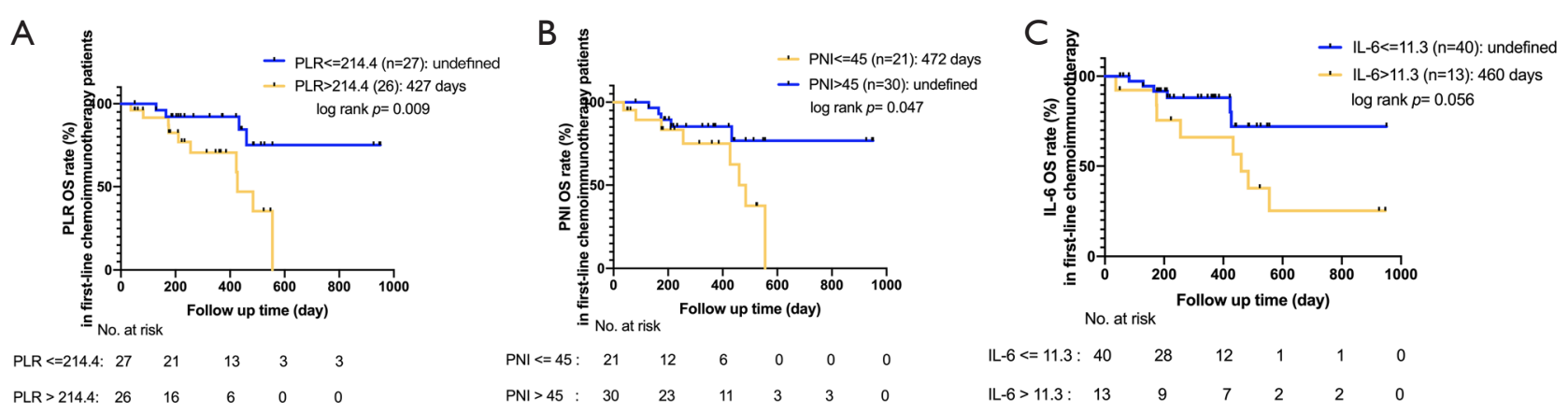

Figure S5 Kaplan-Meier analysis of OS for patients receiving first-line chemoimmunotherapy (n=53) according to the PLR ( $\leq$ median of $214.4 v s .>214.4)$ (A), the PNI ( $\leq 45 v s .>45)$ (B), and the IL-6 ( $\leq 11.3 v s .>11.3)(\mathrm{C})$. The P values were calculated with the log rank test. OS, overall survival; PLR, platelet-to-lymphocyte ratio; PNI, prognostic nutritional index; IL-6, interleukin-6.

Table S8 Multivariate analyses of PFS and OS for patients receiving chemoimmunotherapy for first-line therapy ( $\mathrm{n}=53$, with 51 patient's data available)

\begin{tabular}{|c|c|c|c|c|c|c|c|}
\hline Variable & Category & \multicolumn{3}{|c|}{ PFS } & \multicolumn{3}{|c|}{ os } \\
\hline PLR & $>214.4$ & 1.090 & $0.322-3.690$ & 0.890 & 2.805 & $0.586-13.433$ & 0.197 \\
\hline PNI & $>45$ & 0.887 & $0.252-3.122$ & 0.852 & 0.926 & $0.188-4.552$ & 0.924 \\
\hline IL6 & $>11.3$ & 2.215 & $0.842-5.831$ & 0.107 & 2.354 & $0.644-8.609$ & 0.196 \\
\hline
\end{tabular}

PFS, progression-free survival; OS, overall survival; HR, hazard ratio; CI, confidence interval; PLR, platelet-to-lymphocyte ratio; PNI, prognostic nutritional index; IL-6, interleukin-6. 
Table S9 Changes between pretreatment and on-treatment peripheral blood parameters' associated risk of best overall response

\begin{tabular}{|c|c|c|c|c|c|}
\hline Variables & & CR/PR $(n=27)$ & $S D(n=27)$ & $\mathrm{PD}(\mathrm{n}=5)$ & $P$ value \\
\hline \multicolumn{6}{|l|}{ Blood routine test } \\
\hline \multirow[t]{3}{*}{ ALC } & Q1 & $6(22.2 \%)$ & 7 (25.9\%) & $1(20.0 \%)$ & 0.233 \\
\hline & Q2 & $10(37.0 \%)$ & $5(18.5 \%)$ & $1(20.0 \%)$ & \\
\hline & Q3 & $5(18.5 \%)$ & $5(18.5 \%)$ & $3(60.0 \%)$ & \\
\hline \multirow[t]{4}{*}{ NLR } & Q1 & 7 (25.9\%) & $6(22.2 \%)$ & $2(40.0 \%)$ & 0.854 \\
\hline & Q2 & $7(25.9 \%)$ & $6(22.2 \%)$ & $1(20.0 \%)$ & \\
\hline & Q3 & 7 (25.9\%) & $8(29.6 \%)$ & 0 & \\
\hline & Q4 & $6(22.2 \%)$ & $7(25.9 \%)$ & $2(40.0 \%)$ & \\
\hline \multirow{2}{*}{ AEC } & Q3 & $3(11.1 \%)$ & $8(29.6 \%)$ & $1(20.0 \%)$ & \\
\hline & Q4 & $7(25.9 \%)$ & $5(18.5 \%)$ & $2(40.0 \%)$ & \\
\hline \multirow[t]{4}{*}{ PLR } & Q1 & $8(29.6 \%)$ & $6(22.2 \%)$ & $1(20.0 \%)$ & 0.451 \\
\hline & Q2 & $8(29.6 \%)$ & $6(22.2 \%)$ & 0 & \\
\hline & Q3 & $5(18.5 \%)$ & 9 (33.3\%) & $1(20.0 \%)$ & \\
\hline & Q4 & $6(22.2 \%)$ & $6(22.2 \%)$ & $3(60.0 \%)$ & \\
\hline Lymphocyte subsets test & & $\mathrm{N}=21$ & $\mathrm{~N}=21$ & $\mathrm{~N}=4$ & \\
\hline B lymphocyte & Q1 & $6(28.6 \%)$ & $6(28.6 \%)$ & 0 & 0.549 \\
\hline \multirow{2}{*}{ NK cell } & Q3 & $6(28.6 \%)$ & 4 (19.0\%) & $1(25.0 \%)$ & \\
\hline & Q4 & $3(14.3 \%)$ & 7 (33.3\%) & $1(25.0 \%)$ & \\
\hline \multirow[t]{4}{*}{ CD4 ${ }^{+}$Iymphocyte } & Q1 & $6(28.6 \%)$ & $6(28.6 \%)$ & 0 & 0.043 \\
\hline & Q2 & $5(23.8 \%)$ & $3(14.3 \%)$ & $4(100.0 \%)$ & \\
\hline & Q3 & $5(23.8 \%)$ & $6(28.6 \%)$ & 0 & \\
\hline & Q4 & $5(23.8 \%)$ & $6(28.6 \%)$ & 0 & \\
\hline \multirow[t]{4}{*}{$\mathrm{CD}^{+}$lymphocyte } & Q1 & $6(28.6 \%)$ & 5 (23.8\%) & $1(25.0 \%)$ & 0.986 \\
\hline & Q2 & $6(28.6 \%)$ & $4(19.0 \%)$ & $1(25.0 \%)$ & \\
\hline & Q3 & $5(23.8 \%)$ & $6(28.6 \%)$ & $1(25.0 \%)$ & \\
\hline & Q4 & $4(19.0 \%)$ & $6(28.6 \%)$ & $1(25.0 \%)$ & \\
\hline
\end{tabular}

Table S9 (continued) 
Table S9 (continued)

\begin{tabular}{|c|c|c|c|c|c|}
\hline Variables & & CR/PR $(n=27)$ & $S D(n=27)$ & $\mathrm{PD}(\mathrm{n}=5)$ & $P$ value \\
\hline \multirow{3}{*}{ LDH } & Q1 & 6 (35.3\%) & 3 (18.8\%) & 1 (25.0\%) & 0.747 \\
\hline & Q2 & $4(23.5 \%)$ & 4 (25.0\%) & $1(25.0 \%)$ & \\
\hline & Q3 & 5 (29.4\%) & 3 (18.8\%) & $1(25.0 \%)$ & \\
\hline \multirow[t]{4}{*}{ IL-6 } & & $\mathrm{N}=27$ & $\mathrm{~N}=27$ & $\mathrm{~N}=5$ & \\
\hline & Decrease & $13(48.1 \%)$ & 8 (29.6\%) & 2 (40.0\%) & 0.734 \\
\hline & Stable & 9 (33.3\%) & $13(48.1 \%)$ & 2 (40.0\%) & \\
\hline & Increase & 5 (18.5\%) & $6(22.2 \%)$ & $1(20.0 \%)$ & \\
\hline \multirow{2}{*}{ IL-8 } & Stable & $11(45.8 \%)$ & $12(46.2 \%)$ & 1 (20.0\%) & \\
\hline & Increase & $1(4.2 \%)$ & 4 (15.4\%) & 2 (40.0\%) & \\
\hline \multirow[t]{4}{*}{ IL-10 } & & $\mathrm{N}=24$ & $\mathrm{~N}=26$ & $N=5$ & 0.611 \\
\hline & Decrease & $1(4.2 \%)$ & 0 & 0 & \\
\hline & Stable & 22 (91.7\%) & 23 (88.5\%) & 5 (100.0\%) & \\
\hline & Increase & $1(4.2 \%)$ & 3 (11.5\%) & 0 & \\
\hline
\end{tabular}

ALC, absolute lymphocyte count; NLR, neutrophil-lymphocyte ratio; AEC, absolute eosinophils count; PLR, platelet-lymphocyte ratio; LDH, lactate dehydrogenase; IL-6, interleukin-6; IL-8, interleukin-8; IL-10, interleukin-10. 
Table S10 Changes between pretreatment and on-treatment peripheral blood parameters' associated risk of incidence of irAEs

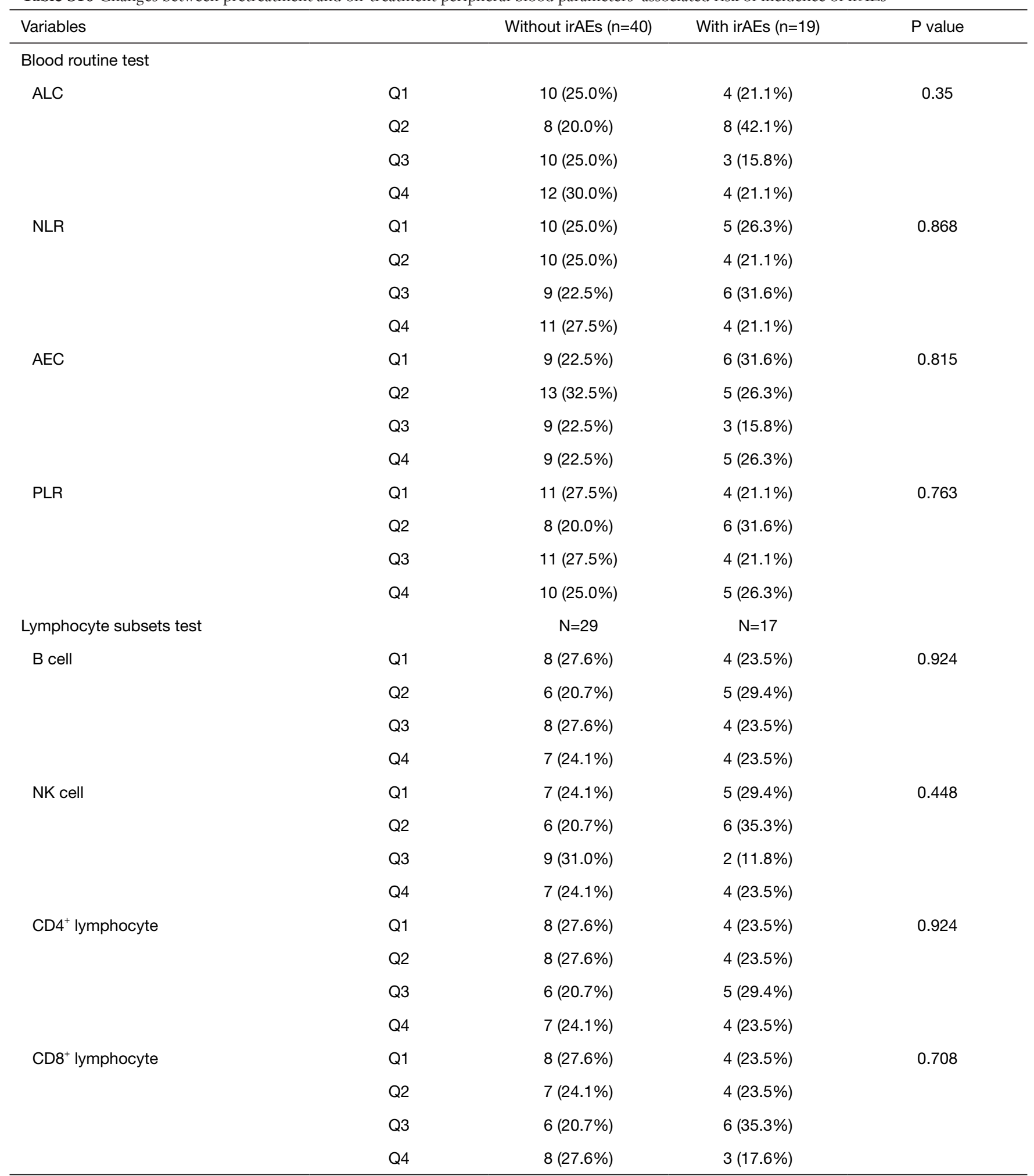

Table S10 (continued) 
Table S10 (continued)

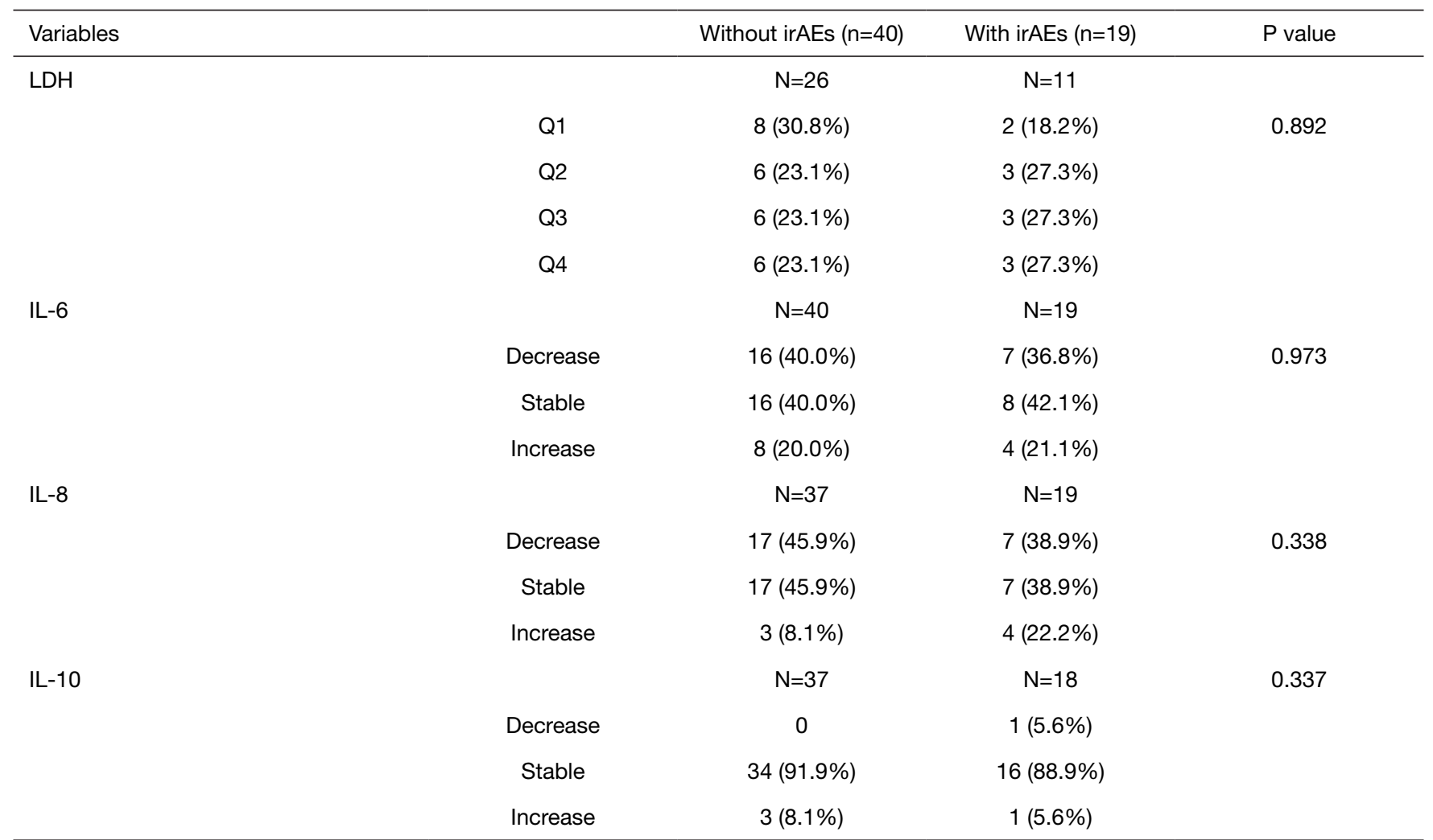

ALC, absolute lymphocyte count; NLR, neutrophil-lymphocyte ratio; AEC, absolute eosinophils count; PLR, platelet-lymphocyte ratio; LDH, lactate dehydrogenase; IL-6, interleukin-6; IL-8, interleukin-8; IL-10, interleukin-10. 
Table S11 Comparison of changes of pretreatment and on-treatment blood parameters (on-treatment results minus pretreatment results) between patients receiving chemoimmunotherapy and patients receiving ICI monotherapy $(\mathrm{n}=59)$

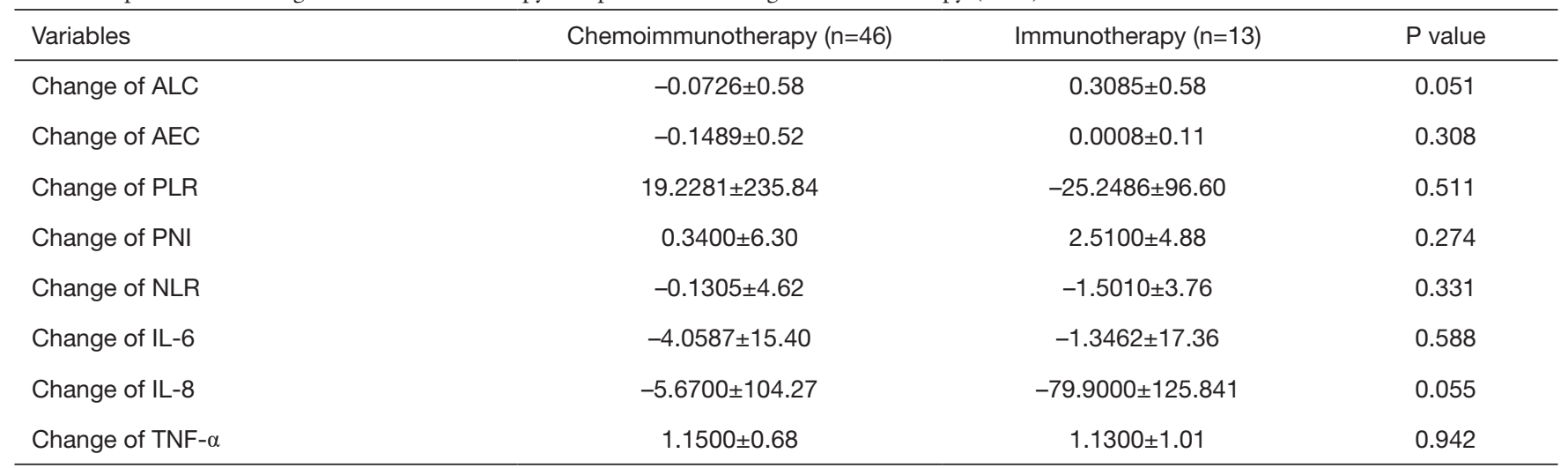

ALC, absolute lymphocyte count; AEC, absolute eosinophils count; PLR, platelet-lymphocyte ratio; PNI, prognostic nutritional index; NLR, neutrophil-lymphocyte ratio; IL-6, interleukin-6; IL-8, interleukin-8; TNF- $\alpha$, tumor necrosis factor $\alpha$. 